\title{
Alteration of thyroid hormone signaling triggers the diabetes-induced pathological growth, remodeling, and dedifferentiation of podocytes
}

\author{
Valentina Benedetti, ${ }^{1}$ Angelo Michele Lavecchia, ${ }^{1}$ Monica Locatelli, ${ }^{1}$ Valerio Brizi, ${ }^{1}$ Daniela Corna, \\ Marta Todeschini, ${ }^{1}$ Rubina Novelli, ${ }^{1}$ Ariela Benigni, ${ }^{1}$ Carlamaria Zoja, ${ }^{1}$ Ciuseppe Remuzzi, ${ }^{1,2}$ \\ and Christodoulos Xinaris'
}

1Istituto di Ricerche Farmacologiche Mario Negri IRCCS, Centro Anna Maria Astori, Science and Technology Park Kilometro Rosso, Bergamo, Italy. ${ }^{2}$ L. Sacco Department of Biomedical and Clinical Sciences, University of Milan, Milan, Italy.

\begin{abstract}
Thyroid hormone (TH) signaling is a universal regulator of metabolism, growth, and development. Here, we show that TH-TH receptor (TH-TR) axis alterations are critically involved in diabetic nephropathy-associated (DN-associated) podocyte pathology, and we identify TR $\alpha 1$ as a key regulator of the pathogenesis of DN. In ZSF1 diabetic rats, $\mathrm{T}_{3}$ levels progressively decreased during DN, and this was inversely correlated with metabolic and renal disease worsening. These phenomena were associated with the reexpression of the fetal isoform TR 1 in podocytes and parietal cells of both rats and patients with DN and with the increased glomerular expression of the TH-inactivating enzyme deiodinase 3 (DIO3). In diabetic rats, TR 1 1-positive cells also reexpressed several fetal mesenchymal and damage-related podocyte markers, while glomerular and podocyte hypertrophy was evident. In vitro, exposing human podocytes to diabetes milieu typical components markedly increased TR 1 and DIO3 expression and induced cytoskeleton rearrangements, adult podocyte marker downregulation and fetal kidney marker upregulation, the maladaptive cell cycle induction/arrest, and TRo1-ERK1/2-mediated hypertrophy. Strikingly, $\mathrm{T}_{3}$ treatment reduced $\mathrm{TR} \alpha 1$ and $\mathrm{DIO} 3$ expression and completely reversed all these alterations. Our data show that diabetic stress induces the TH-TR $\alpha 1$ axis to adopt a fetal ligand/receptor relationship pattern that triggers the recapitulation of the fetal podocyte phenotype and subsequent pathological alterations.
\end{abstract}

Authorship note: V. Benedetti and AML contributed equally to this work.

Conflict of interest: The authors have declared that no conflict of interest exists.

Copyright: () 2019, American Society for Clinical Investigation.

Submitted: May 13, 2019

Accepted: August 13, 2019

Published: September 19, 2019.

Reference information: /CI Insight. 2019;4(18):e130249.

https://doi.org/10.1172/jci.

insight.130249.

\section{Introduction}

Thyroid hormone (TH) signaling is a universal regulator of the metabolism, growth, and development. Highly conserved in the animal kingdom, TH signaling played a crucial role in the colonization of land by organisms, the evolution of complex organs, epimorphic regeneration, metamorphosis, endothermy, and hibernation (1-3). The active form of $\mathrm{TH}$, L-triiodothyronine $\left(\mathrm{T}_{3}\right)$, acts by regulating gene transcription through the binding to its nuclear receptors, TH receptor (TR) $\alpha$ and $\beta$ (4). TRs have distinct expression patterns and distinct functional roles during fetal and adult life that are contingent on their liganded states. TR $\alpha 1$ regulates larval transition, amphibian metamorphosis and morphogenesis, and tissue maturation in the mammalian embryo (5-7). It is the predominant form in developing organs, such as the heart (8) and brain (9), while TR $\beta 1$ is highly expressed during adulthood. During fetal life, $\mathrm{T}_{3}$ levels are low, and unliganded TR $\alpha 1$ (aporeceptor) represses the transcription of target adult genes. After birth, $\mathrm{T}_{3}$ levels increase, and liganded TR $\alpha 1$ (holoreceptor) triggers different gene expression programs, promoting postnatal development and maturation. The low availability of $\mathrm{T}_{3}$ - which is indispensable for the normal development of the fetus — is guaranteed by the TH-inactivating enzyme deiodinase 3 (DIO3). DIO3 is expressed abundantly in fetal tissues to protect the fetus against high maternal $\mathrm{T}_{3}$ levels, thus preventing precocious tissue maturation (10).

In diabetes, the fetal profile of low $\mathrm{T}_{3}$ levels recurs: diabetic patients have significantly lower $\mathrm{T}_{3}$ plasma levels (11) and a high prevalence of thyroid dysfunction compared with the healthy population, while hypothyroidism — both clinical and subclinical — is the most common diabetes-associated disorder $(12,13)$. 
Table 1. Systemic parameters in lean and ZSF1 rats at 4 and 6 months of age

\begin{tabular}{|l|c|c|c|c|c|}
\hline Group & \multicolumn{2}{|c|}{ Body weight (g) } & \multicolumn{2}{c|}{$\begin{array}{c}\text { Proteinuria (mg/24 h) } \\
\text { Glomerulosclerosis } \\
\text { index }\end{array}$} \\
\hline & 4 months & 6 months & 4 months & 6 months & 6 months \\
\hline Lean rats $(n=4)$ & $440 \pm 7$ & $489 \pm 12$ & $17.7 \pm 0.9$ & $19.2 \pm 1.6$ & $0.01 \pm 0.00$ \\
\hline ZSF1 rats $(n=8)$ & $574 \pm 9^{\mathrm{A}}$ & $695 \pm 20^{\mathrm{A}}$ & $229.3 \pm 34.6^{\mathrm{B}}$ & $511.2 \pm 71.3^{\mathrm{A}}$ & $0.53 \pm 0.08^{\mathrm{C}}$ \\
\hline
\end{tabular}

Data are expressed as mean \pm SEM and were analyzed by Student's $t$ test. ${ }^{A} P<0.001,{ }^{B} P<0.01$, vs. control lean.

Several clinical studies have also shown that, in diabetic patients, thyroid dysfunction and low $\mathrm{T}_{3}$ levels are strongly associated with worse renal clinical outcomes and increased mortality (14-16). Diabetic nephropathy $(\mathrm{DN})$ is one of the most common complications of diabetes mellitus and the leading cause of end-stage renal disease worldwide. Primary hallmarks of DN are podocyte dedifferentiation, hypertrophy, maladaptive proliferation, and apoptosis (17). Podocytes are particularly vulnerable to the noxious stimuli of diabetic milieu $(18,19)$, and, since they cannot proliferate, podocyte damage and loss result in the impairment of the integrity and function of the glomerular filtering barrier and the subsequent loss of proteins into the urine (17). In response to the various stressful stimuli (including high glucose, free radicals, mechanical stress), podocytes reenter the cell cycle, increase in size, and frequently undergo mitosis with nuclear division but do not complete cytokinesis $(20,21)$, generating aneuploid podocytes. These rapidly detach and die in a process called mitotic catastrophe (22). Surviving podocytes undergo hypertrophy and dedifferentiation, which is characterized by structural remodeling, the downregulation of terminally differentiated podocyte markers, the concurrent reactivation of developmental pathways, and the acquisition of mesenchymal features (23-28). This adoption of a fetal phenotype results in overall structural and metabolic remodeling of the glomerulus, with detrimental consequences for the kidney's filtering functions. Although these phenomena have been observed in $\mathrm{DN}$, the etiogenesis remains poorly understood.

Considering (a) the biological roles that TH signaling has in organ development and maturation, (b) the changes in TH availability in diabetes, and (c) the concomitant reactivation of developmental pathways in diabetic podocytes, we hypothesized that the TH-TR $\alpha 1$ axis plays a key role in the pathogenesis of DN by triggering an inappropriate reactivation of podocyte developmental programs and subsequent pathological growth and remodeling. To verify this hypothesis, we studied changes in TH signaling in ZSF1 diabetic rats and diabetic patients, and in vitro models of $\mathrm{DN}$, as well as the possible underlying molecular pathways. Our findings demonstrate that in DN the TH-TR signaling adopts a ligand/receptor relationship profile that resembles that of early embryonic kidney development: high TR $\alpha 1$ in low levels of $\mathrm{T}_{3}$ may act as aporeceptor to trigger early developmental pathways (as it does during normal development) that are responsible for the "fetal-like" pathomorphological changes observed in diabetic podocytes. Moreover, our data indicate that diabetic stress-induced alterations in podocytes are manifestations of an imperfect/maladaptive partial recapitulation of early developmental steps and identify TR $\alpha 1$ as a possible therapeutic target for the treatment of DN.

\section{Results}

Diabetes induces the fetal pattern of renal TH signaling that may be involved in the progression of nephropathy. To examine whether $\mathrm{TH}$ signaling is associated with the progression of $\mathrm{DN}$, we analyzed changes in circulating $\mathrm{T}_{3}$ levels and the relationships between $\mathrm{T}_{3}$ levels and parameters of metabolic or renal disease in ZSF1 rats (Table 1). At 3 months of age - at the beginning of the study $-\mathrm{T}_{3}$ levels started to decline in ZSF1 rats and dropped to significantly lower levels than in lean controls as the disease progressed (Figure 1A). The $\Delta$ between the $\mathrm{T}_{3}$ levels of ZSF1 rats at 4 and 6 months of age, in particular, was inversely correlated with $\Delta$ of body weight $\left(\mathrm{R}^{2}=0.8754\right.$ and $\left.P=0.0061\right)$ and with $\Delta$ of proteinuria levels $\left(\mathrm{R}^{2}=0.7822\right.$ and $\left.P=0.0193\right)$ at the same ages. $\mathrm{T}_{3}$ levels were also inversely correlated with the glomerulosclerosis index $\left(\mathrm{R}^{2}=0.6154\right.$ and $\left.P=0.0212\right)$ (Figure 1 , B-D), indicating that TH signaling was possibly involved in the progression and related to the severity of DN.

We then investigated whether $\mathrm{T}_{3}$ level decrements were associated with changes in TRs' expression. Due to a lack of data in kidneys, we first evaluated TR expression patterns in renal tissues of rats at various developmental stages. TR $\alpha 1$ was highly expressed in rat embryonic kidneys, while it declined significantly postnatally (Figure 2A); TR $\beta 1$ remained unchanged throughout development (data not shown). Then, we analyzed TR $\alpha 1$ and TR $\beta 1$ protein expression in kidney specimens from diabetic ZSF1 rats at 6 months of age, when overt 
A

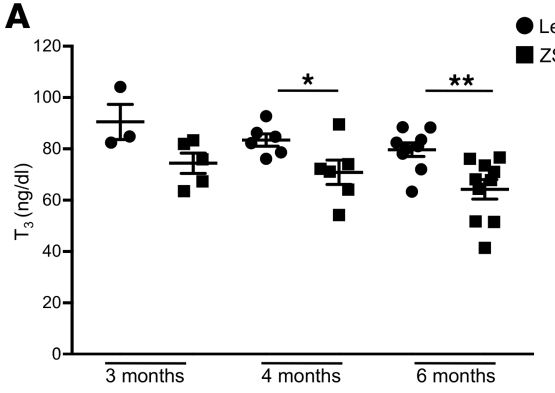

C

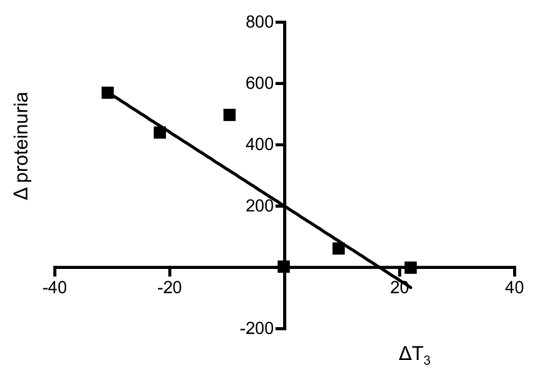

B

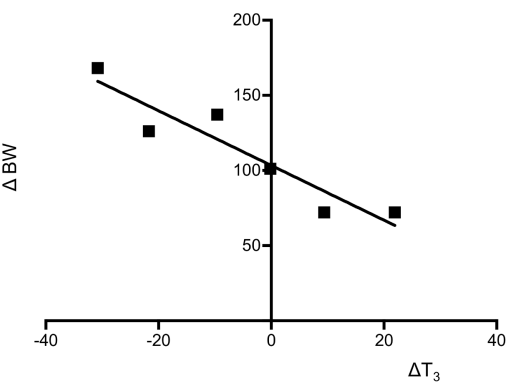

D

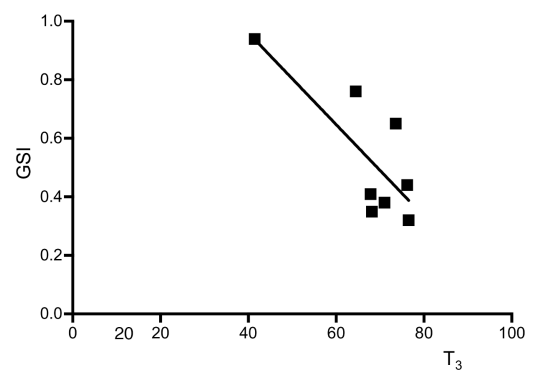

Figure 1. Changes in $\mathrm{T}_{3}$ levels and correlation of $\mathrm{T}_{3}$ with obesity and renal function parameters in diabetic rats. (A) Serum $T_{3}$ levels (ng/dl) declined progressively in ZSF1 rats at 3, 4, and 6 months of age compared with control rats. Data are expressed as mean $\pm \mathrm{SEM} .{ }^{*} P<0.05,{ }^{* *} P<0.01$, unpaired Student's $t$ test. $n=3-10$ rats per group. (B-D) Relationship between $T_{3}$ levels and parameters of obesity or renal disease in ZFS1 rats at 6 months of age. $\Delta \mathrm{T}_{3}$ levels $(\mathrm{ng} / \mathrm{dl})$ between 6 and 4 months of age inversely correlated with $(\mathrm{B}) \Delta \mathrm{BW}(\mathrm{g})\left(\mathrm{R}^{2}=0.8754, P=0.0061\right.$, $n=6$ rats) and (C) with $\Delta$ proteinuria levels (mg/day) $\left(R^{2}=0.7822, P=0.0193, n=6\right.$ rats). (D) $T_{3}$ levels negatively correlated with $\mathrm{CSI}$ in ZSF1 rats $\left(\mathrm{R}^{2}=0.6154, P=0.0212, n=8\right.$ rats). $\mathrm{R}^{2}$ values are determined by linear regression. GSI, glomerulosclerosis index.

nephropathy had developed. Western blot analysis revealed that TR $\alpha 1$ expression was 2.95 -fold higher in the kidneys of ZSF1 rats than in lean controls (ZSF1, $17.50 \pm 2.67$ vs. lean, $5.94 \pm 2.5, P<0.05$ ) (Figure 2B). Immunofluorescence analysis showed increased expression of TR $\alpha 1$ in nestin-positive podocytes and parietal cells of diabetic rats, compared with lean controls, where glomerular TR $\alpha 1$ expression was almost absent (Figure 2, C and D). In contrast, TR $\beta 1$ expression was higher in nestin-positive podocytes of lean control rats than in ZSF1 rats (Figure 2, C and D). Analysis of biopsies from patients with DN and control subjects consistently confirmed the TR $\alpha 1$ and TR $\beta 1$ expression pattern observed in diabetic and lean rats, respectively (Figure 2, E and F).

To examine if the $\mathrm{TH}$-inactivating enzyme DIO3 was involved in the alterations in $\mathrm{TH}$ signaling observed in diabetes, we investigated DIO3 expression and localization in the kidneys of ZSF1 and lean rats. DIO3 protein expression was 3.74-fold higher in diabetic rats than in the lean controls (ZSF1, 8.35 \pm 1.26 vs. lean, $2.23 \pm 0.73, P<0.01$ ) (Figure $2 \mathrm{G}$ ); it was expressed extensively in the glomerular tuft, parietal cells, and in podocytes of diabetic rats (Figure 2G, inset, and Supplemental Figure 1A; supplemental material available online with this article; https://doi.org/10.1172/jci.insight.130249DS1), while it was absent in control rats.

These data demonstrate the adoption of the fetal $\mathrm{TH}$ signaling profile (low $\mathrm{T}_{3} /$ high $\mathrm{TR} \alpha 1$ and high DIO3) by kidneys of rats and patients with DN.

TR 1 upregulation is associated with the reactivation of kidney developmental programs during $D N$. To assess whether the fetal ligand/receptor relationship pattern was associated with the reactivation of further developmental pathways, we analyzed the expression of embryonic kidney markers in renal specimens from ZSF1 rats. Western blot analysis showed a significantly increased expression of molecules that are essential for kidney development, such as paired box 2 (Pax2) (29), SIX homeobox 2 (Six2) (30), and glial cell line-derived neurotrophic factor (GDNF) (31) in the kidneys of rats with DN compared with lean rats (Pax2, 2.95-fold; Six2, 1.50-fold; and GDNF, 1.71-fold higher than in controls) (Figure 3, A-C). Immunostaining assays demonstrated that embryonic markers were expressed extensively in the glomerular tuft, in nestin-positive podocytes, parietal cells, and in some tubules of ZSF1 rats. In the glomeruli of lean rats we detected only faint expression of these embryonic markers (Figure 3, A-D). Further investigations 
A

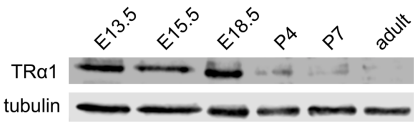

C

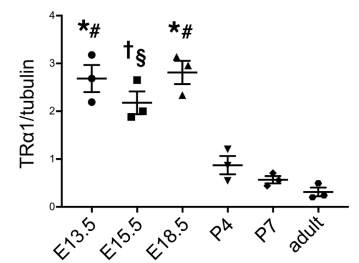

Lean

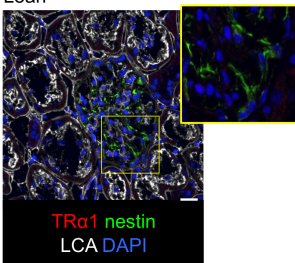

E

Control

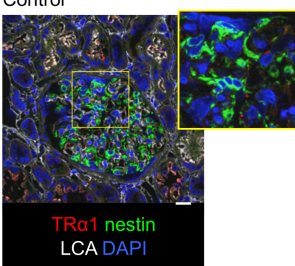

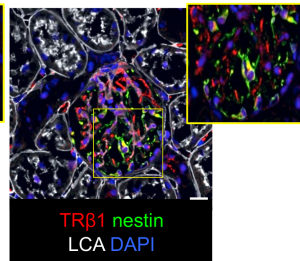

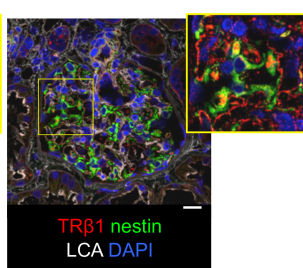

B
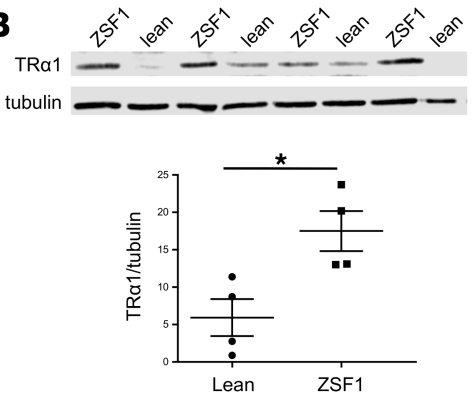

D
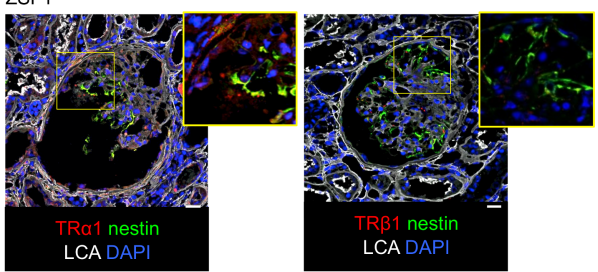

$\mathbf{F}$
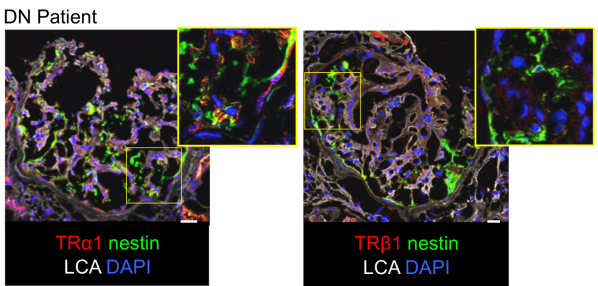

G
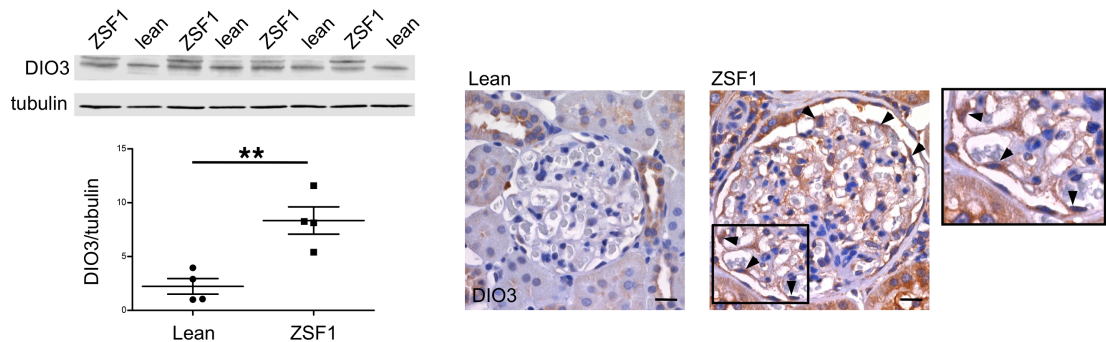

Figure 2. Adoption of fetal expression pattern of TRs and DIO3 during DN. (A) Western blot and densitometric analysis of TR $\alpha 1$ protein levels. TR $\alpha 1$ was highly expressed in rat embryonic kidneys (E13.5-E18.5) and significantly reduced after birth. Data are expressed as mean \pm SEM. ${ }^{*} P<0.0001$ vs. $P 7$ and adult, ${ }^{\dagger} P<0.0005$ vs. adult, ${ }^{\sharp} P<0.0005$ vs. $P 4,{ }^{\circledR} P<0.01$ vs. P4 and P7, 1-way ANOVA corrected with Tukey's post hoc test. $n=20 \mathrm{E} 13.5, n=16 \mathrm{E} 15.5, n=12 \mathrm{E} 18.5, n=3$ postnatal and adult kidneys. (B) TR $\alpha 1$ levels increased in kidneys of ZSF1 rats compared with lean rats at 6 months of age. ${ }^{*} P<0.05$, unpaired Student's $t$ test. $n=4$ rats per group. (C-F) Representative images of TR $\alpha 1$ and TR $\beta 1$ protein stainings in kidney sections from (C) lean and (D) ZSF1 rats and in (E) portions of control human kidney tissue and (F) from renal biopsies of DN patient. TR $\alpha 1$ (red) was expressed in nestin-positive (green) podocytes and parietal cells of ZSF1 rats and DN patients, while it was almost absent in lean rats and control human tissue (C-F, left). Conversely, TR $\beta 1$ (red) was highly expressed in glomerular tuft and podocytes of lean rats and human controls, while it was lower in ZSF1 rats and DN patients (C-F, right). $n=3$ rats and $n=3$ human subjects per group. (G) Western blot and densitometric analysis of DIO3 protein levels (left). DIO3 levels increased in kidneys of ZSF1 rats compared with lean rats. Representative images of DIO3 staining in lean and ZSF1 rat kidneys (right). DIO3 was highly expressed in glomerular tuft, parietal cells, and podocytes of ZSF1 rats (right, arrows). Data are expressed as mean \pm SEM. ${ }^{* *} P<0.01$, unpaired Student's $t$ test. $n=4$ rats per groups. Tubulin protein expression was used as sample loading control. Scale bars: $20 \mu \mathrm{m}$. LCA, Lens culinaris agglutinin.

in serial sections with colocalization analysis showed that most of fetal marker-positive cells were also expressing TR $\alpha 1$, indicating that TR $\alpha 1$ could be involved in their abnormal reactivation (Figure $3, \mathrm{~A}-\mathrm{C}$ ). In line with these findings, we also observed an increased expression of desmin, a mesenchymal marker (32), which colocalized with TR 1 1-positive cells in glomeruli of diabetic rats (Supplemental Figure 1, B and C). Moreover, the expression of the GDNF receptor tyrosine kinase (Ret) and the GDNF family receptor $\alpha$ 1 (GFR $\alpha 1$ ) - proteins that are known to be expressed by podocytes during development and injury (33) 

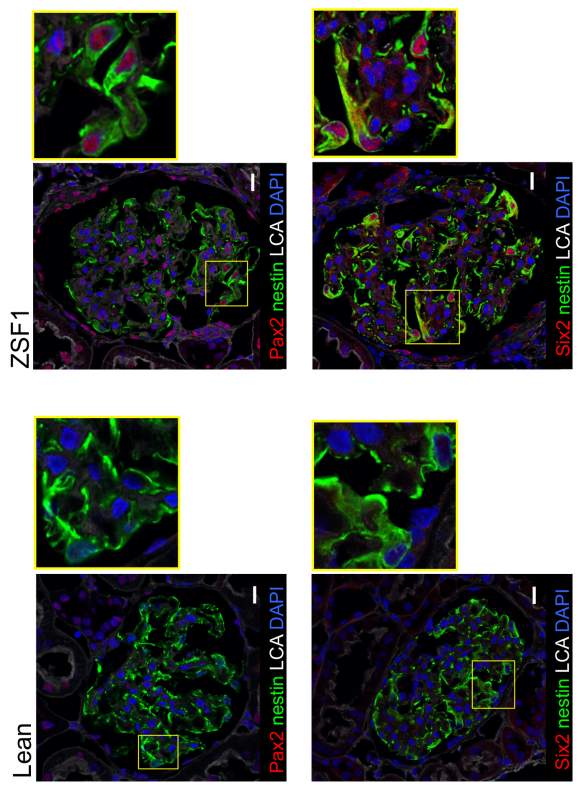

口
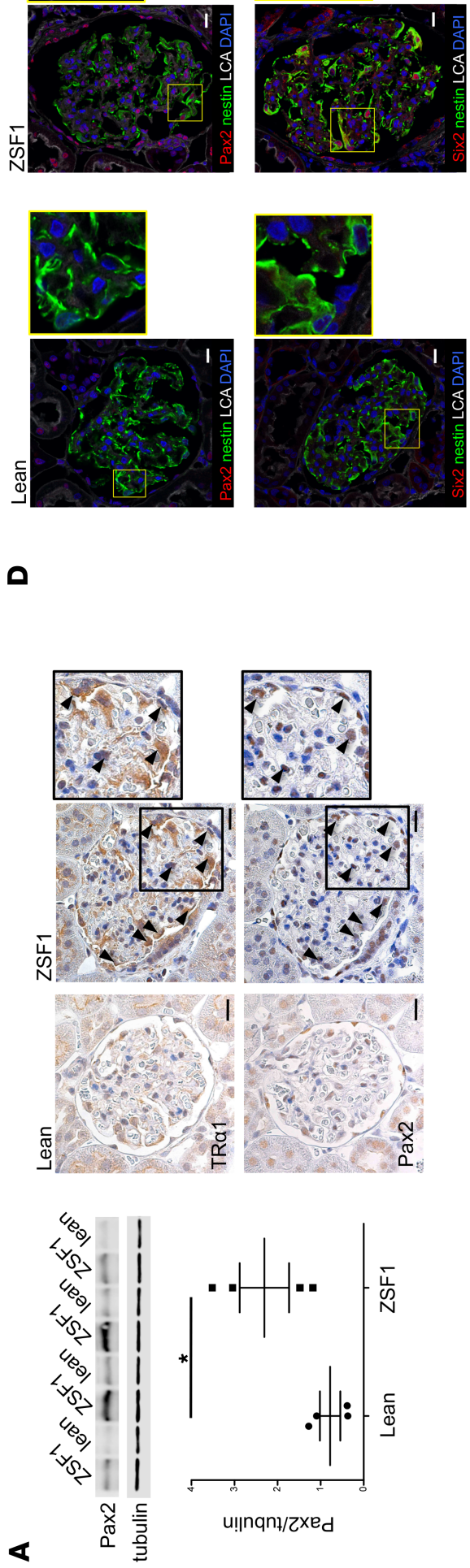
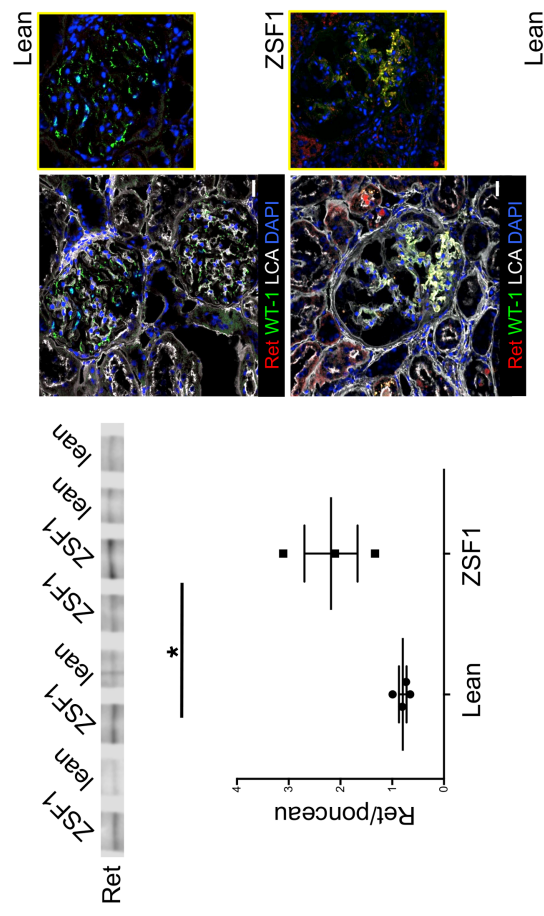

Ш
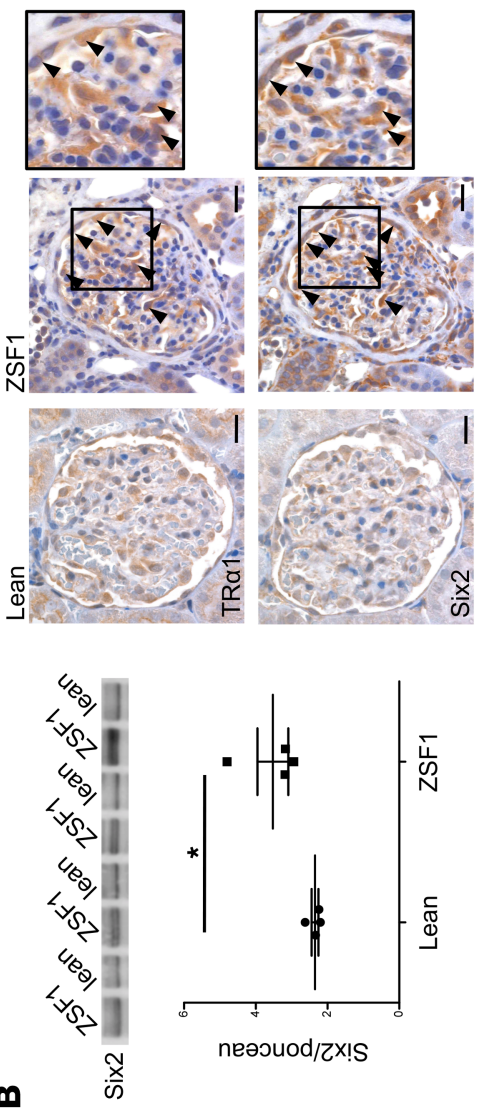
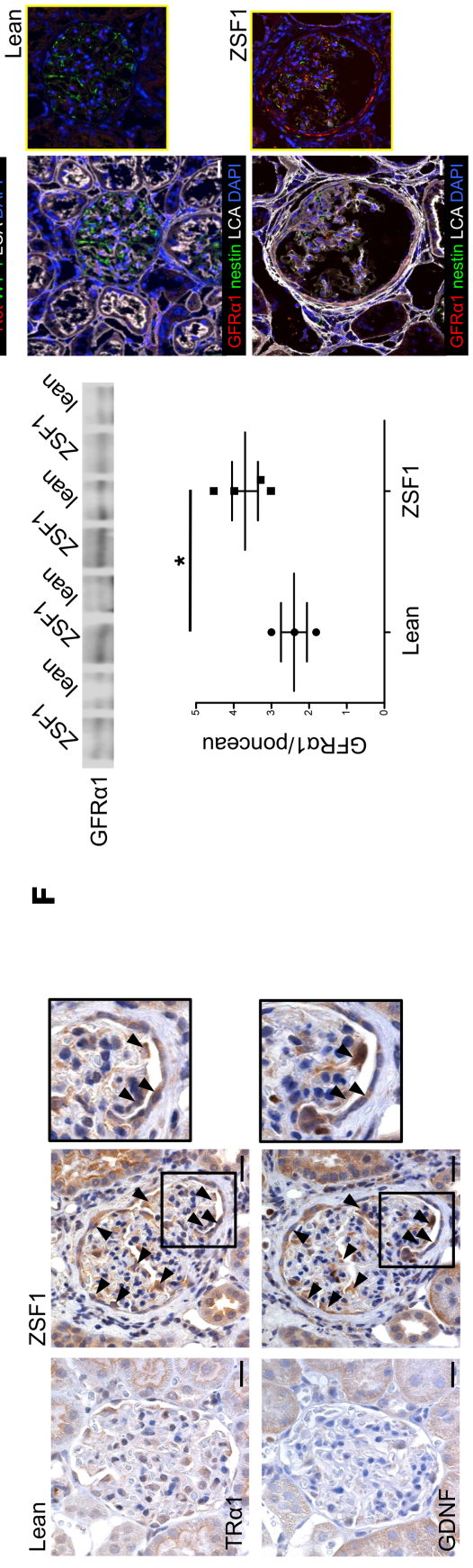

$\boldsymbol{L}$

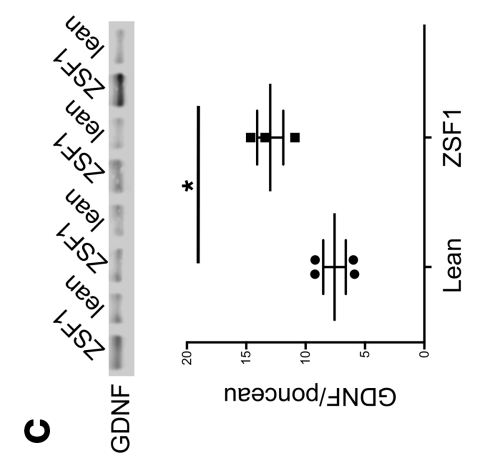


Figure 3. TRa1 reexpression is associated with reactivation of kidney developmental program in diabetic rats. (A-C) Expression of fetal markers in kidneys of ZSF1 and lean rats at 6 months of age. Western blot and densitometric analysis of (A) Pax2, (B) Six2, and (C) GDNF protein levels in ZSF1 rats compared with lean rats (left). Representative images of TR $\alpha 1$ and (A) Pax2, (B) Six2, and (C) GDNF stainings on serial sections in lean and ZSF1 rats (right). Fetal markers were highly expressed in the glomerular tuft and parietal cells of ZSF1 rats. (D) Representative images of nestin-positive podocytes (green) expressing Pax2 (red; top) and Six2 (red; bottom) in kidney sections of diabetic rats. (E and F) Western blot and densitometric analysis of (E) Ret and (F) GFR 1 protein levels in ZSF1 rats compared with control rats (left). Representative images of (E) Ret and WT-1 and (F) GFR 1 and nestin stainings in lean and ZSF1 rats (right). Ret and GFR 1 (red) expression significantly increased in kidneys of ZSF1 rats compared with lean rats. Data are expressed as mean \pm SEM. ${ }^{*} P<0.05$, unpaired Student's $t$ test. $n=3-4$ rats per group. Tubulin protein expression $(\mathbf{A})$ or ponceau red staining $(\mathbf{B}, \mathbf{C}, \mathbf{E}$, and $\mathbf{F})$ was used as sample loading control. Scale bars: $20 \mu \mathrm{m}$. LCA, Lens culinaris agglutinin.

- increased markedly in ZSF1 rats (Ret, 2.73-fold, and GFR 1 1, 1.54-fold higher than in the lean control) and were localized in podocytes and parietal cells (Figure 3, E and F). To examine whether the combined alterations in $\mathrm{TH}$ signaling and reactivation of fetal kidney genes is common between different diabetes models and species, we studied stored samples from ZDF rats (tissue and sera) and BTBR ${ }^{o b / o b}$ and streptozotocin-treated C57BL/6 mice (tissues). The results showed that the serum $\mathrm{T}_{3}$ levels were significantly lower in ZDF rats than in lean controls and that TR 1 , DIO3, Six2, and Pax2 were higher in the glomeruli of all diabetic animals (Supplemental Figure 2).

Together, these data indicate that diabetes induces a partial reactivation of the kidney developmental program in the glomerulus of ZSF1 rats, in which the TH-TR $\alpha 1$ axis is involved.

Hypertrophic and polynucleated podocytes are detected in ZSF1 rats. Glomerular and podocyte hypertrophy is a hallmark of DN and has been extensively documented in patients with $\mathrm{DN}$ and in some rodent models of diabetes $(34,35)$; however, in ZSF1 rats, podocyte hypertrophy has not been described yet. Thus, 6-month-old ZSF1 and lean rats were analyzed for glomerular volume; podocyte number and density, which was defined as the ratio between podocyte number and glomerular volume; and individual podocyte volume, which was obtained by the ratio between total glomerular podocyte volume and podocyte number. The overall glomerular volume was significantly higher (ZSF1, $1.84 \pm 0.16 \mu \mathrm{m}^{3} \times 10^{6}$ vs. lean, $1.38 \pm 0.07 \mu \mathrm{m}^{3} \times 10^{6}, P<0.05$ ) (Figure $4 \mathrm{~A}$ ), while the number of WT-1 positive podocytes was significantly lower (ZSF1 $163 \pm 7$ vs. lean $213 \pm 13, P<0.01$ ) (Figure 4B) in ZSF1 rats compared with lean rats. As a result, podocyte density was markedly reduced in ZSF1 rats compared with lean controls (ZSF1, $9.01 \pm 0.61$ cell number $/ 10^{5} \mu \mathrm{m}^{3}$ glomerular volume vs. lean, $15.60 \pm 1.26$ cell number $/ 10^{5} \mu \mathrm{m}^{3}$ glomerular volume, $P<0.01$ ) (Figure $4 \mathrm{C}$ ). We then calculated the total podocyte volume per glomerulus, measuring the percentage of area that was positive for Glepp1, a podocyte apical cell membrane marker. Although podocyte number was significantly reduced, we surprisingly found that Glepp1 expression was only moderately decreased in ZSF1 rats compared with lean rats (ZSF1, 36.7\% $\pm 2.9 \%$ vs. lean, $43.6 \%$ $\pm 1.5 \%$ ), while the Glepp1-positive tuft volume, which was estimated by multiplying the percentage of Glepp1-positive area and the glomerular volume, did not change (ZSF1 $6.9 \pm 1.2 \mu \mathrm{m}^{3} \times 10^{5}$ vs. lean 6.0 $\left.\pm 0.3 \mu \mathrm{m}^{3} \times 10^{5}\right)$. As explanation of these data, individual podocyte volume was significantly increased in ZSF1 rats compared with lean controls (ZSF1, $3.87 \pm 0.29 \mu \mathrm{m}^{3} \times 10^{3}$ vs. lean, $2.9 \pm 0.3 \mu \mathrm{m}^{3} \times 10^{3}, P$ $<0.05$ ) (Figure 4, D and E), highlighting the presence of significant podocyte hypertrophy. Moreover, we occasionally observed hypertrophic podocytes with 2 nuclei (Figure 4D, inset, arrows).

These data clearly document the presence of both glomerular and podocyte hypertrophy in ZSF1 diabetic rats and that this hypertrophy was associated with the occurrence of polynucleated podocytes.

TH signaling alterations drive podocyte cytoskeleton rearrangement and dedifferentiation in vitro that are total ly reversed by $T_{3}$ treatment. To study the role of TR $\alpha 1$ in inducing the molecular and phenotypical changes observed in vivo in podocytes that are damaged by DN, we cultured human immortalized podocytes in the presence of high glucose concentration and reactive oxygen species $\left(\mathrm{H}_{2} \mathrm{O}_{2}\right)$ - key components of the diabetic milieu - for 9 days. Immunofluorescence analysis showed that some untreated cells expressed TR $\alpha 1$ in the nuclear and perinuclear areas. High glucose and $\mathrm{H}_{2} \mathrm{O}_{2}$ treatment markedly increased cell size and TR 1 expression compared with controls; in large cells, TR $\alpha 1$ was highly expressed in the cytoplasm (Figure 5A, top, and Figure 5B). Polynucleated cells were visible after high glucose treatment (Figure 5C, asterisks).

According to the dual-function model for the role of TRs in postembryonic development of some mammalian organs (36) and in the metamorphosis of amphibians, TR 1 can (a) repress target genes that are involved in differentiation to allow tissue/organ growth in the absence of $\mathrm{TH}$ or (b) bind $\mathrm{TH}$ and induce the previously repressed genes and others to initiate tissue/organ maturation following $\mathrm{TH}$ release into the circulation. Based on this knowledge, we tested whether adding $\mathrm{T}_{3}$ to the environment could induce 
A

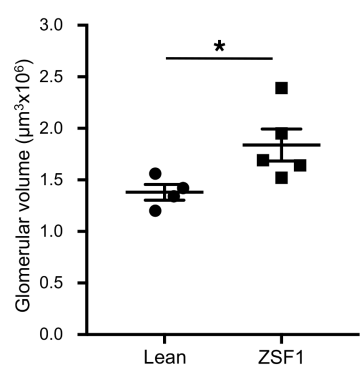

D
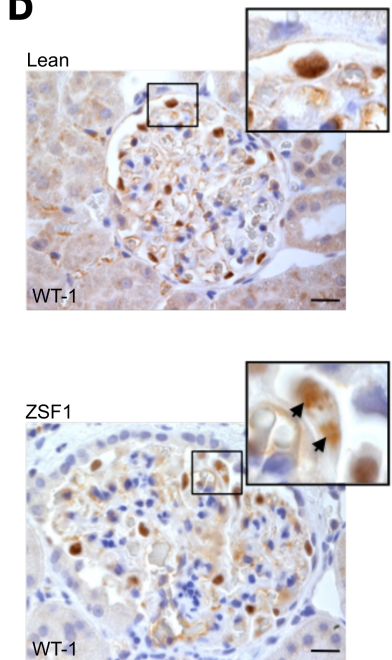

B
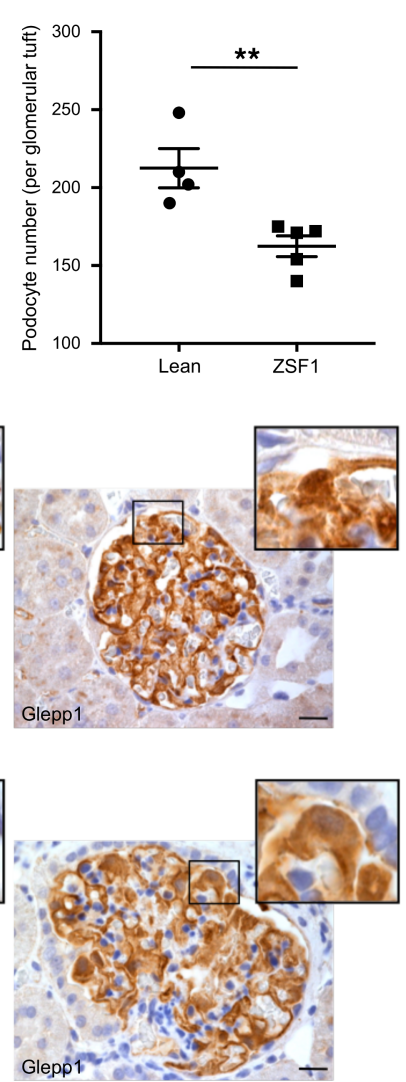

C

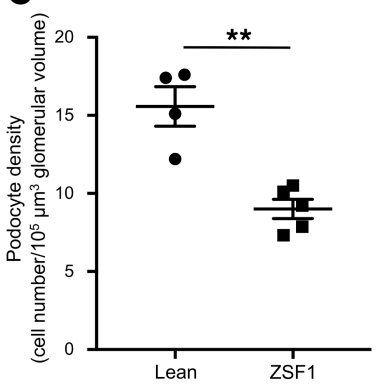

$\mathbf{E}$

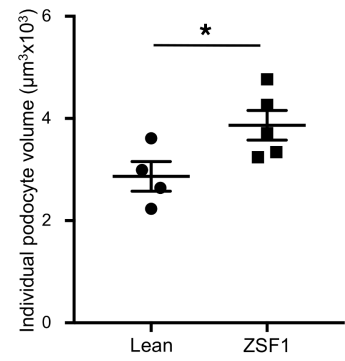

Figure 4. Glomerular volume and podocyte depletion and hypertrophy in ZSF1 rats. (A and B) Quantification of glomerular volume and WT-1-positive podocytes in lean and ZSF1 rats at 6 months of age. Diabetic rats exhibited (A) increased glomerular volume, (B) decreased number of podocytes per glomerulus, and (C) lower podocyte density compared with lean controls. (D) Representative images of WT-1 and Glepp1 staining on serial sections in lean and ZSF1 rats. Insets show enlarged images of podocytes and highlight a polynucleated podocyte. (E) Quantification of individual podocyte volume in lean and ZSF1 rats. Diabetic rats had significantly greater podocyte volume compared with lean controls. Data are expressed as mean \pm SEM. ${ }^{*} P<0.05$; ${ }^{* *} P<0.01$, unpaired Student's $t$ test. $n=4-5$ rats per group. Scale bars: $20 \mu \mathrm{m}$.

TR $\alpha 1$ to reverse the injury-induced pathological reactivation of the podocyte developmental program, thus restoring a more mature podocyte phenotype. We exposed human podocytes to high glucose or $\mathrm{H}_{2} \mathrm{O}_{2}$ for 3 days and then to each stimulus in combination with $\mathrm{T}_{3}$ for an additional 6 days. Treatment with $\mathrm{T}_{3}$ reduced cell size and shifted the TR $\alpha 1$ localization from the cytoplasm to the nuclear and perinuclear areas of both glucose- and $\mathrm{H}_{2} \mathrm{O}_{2}$-stimulated podocytes (Figure $5 \mathrm{~A}$, bottom). Western blot analysis showed that both high glucose ( $1.53 \pm 0.06$ vs. control, $P<0.0001)$ and $\mathrm{H}_{2} \mathrm{O}_{2}(1.80 \pm 0.12$ vs. control, $P<0.0001)$ increased TR $\alpha 1$, while $\mathrm{T}_{3}$ restored $\mathrm{TR} \alpha 1$ to control levels (glucose $+\mathrm{T}_{3}, 0.92 \pm 0.07, \mathrm{H}_{2} \mathrm{O}_{2}+\mathrm{T}_{3}, 1.13 \pm 0.11$ ) (Figure 5D).

In DN, foot process effacement and cell retraction are observed in podocytes, as a consequence of cytoskeletal rearrangements. To investigate whether the restoration of the adult ligand/receptor relationship pattern - obtained through the administration of $\mathrm{T}_{3}$ - could reverse the morphological changes in podocytes damaged by high glucose and oxidative stress, we analyzed changes in the actin-based cytoskeleton of podocytes exposed to high glucose or $\mathrm{H}_{2} \mathrm{O}_{2}$ treated or not with $\mathrm{T}_{3}$. As expected, untreated podocytes showed F-actin fibers arranged in parallel and extended across the entire cell body, while exposure to high glucose or $\mathrm{H}_{2} \mathrm{O}_{2}$ induced F-actin rearrangement at the cell periphery in $42.92 \% \pm 3.67 \%$ and $36.96 \% \pm 2.85 \%$ of podocytes, respectively, compared with $3.53 \% \pm 1.31 \%$ of untreated cells. Notably, treatment with $\mathrm{T}_{3}$ induced F-actin redistribution and alignment, reestablishing an actin-based cytoskeleton similar to that observed in control cells (glucose $+\mathrm{T}_{3}$, $9.86 \% \pm 1.56 \% ; \mathrm{H}_{2} \mathrm{O}_{2}+\mathrm{T}_{3}, 9.84 \% \pm 1.95 \%$ ) (Figure $5 \mathrm{E}$ ).

Moreover, $\mathrm{T}_{3}$ treatment in human podocytes (a) restored the expression of the adult podocyte markers $\alpha$-actinin4 (glucose $+\mathrm{T}_{3}, 1.04 \pm 0.17$ vs. glucose, $0.48 \pm 0.04, P<0.01 ; \mathrm{H}_{2} \mathrm{O}_{2}+\mathrm{T}_{3}, 1.12 \pm 0.10$ vs. $\mathrm{H}_{2} \mathrm{O}_{2}$, 
A
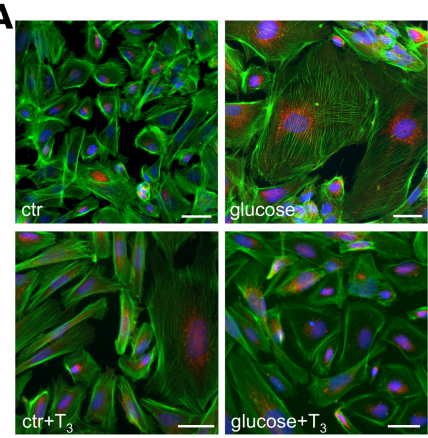

E

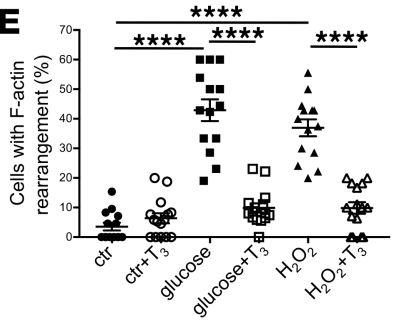

H
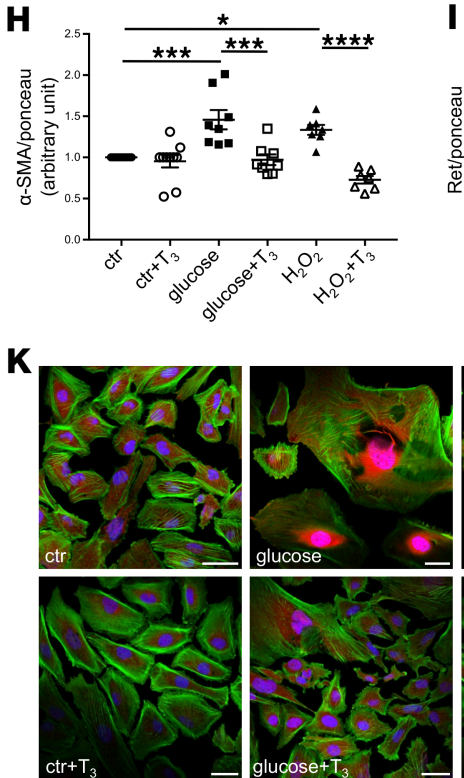

I
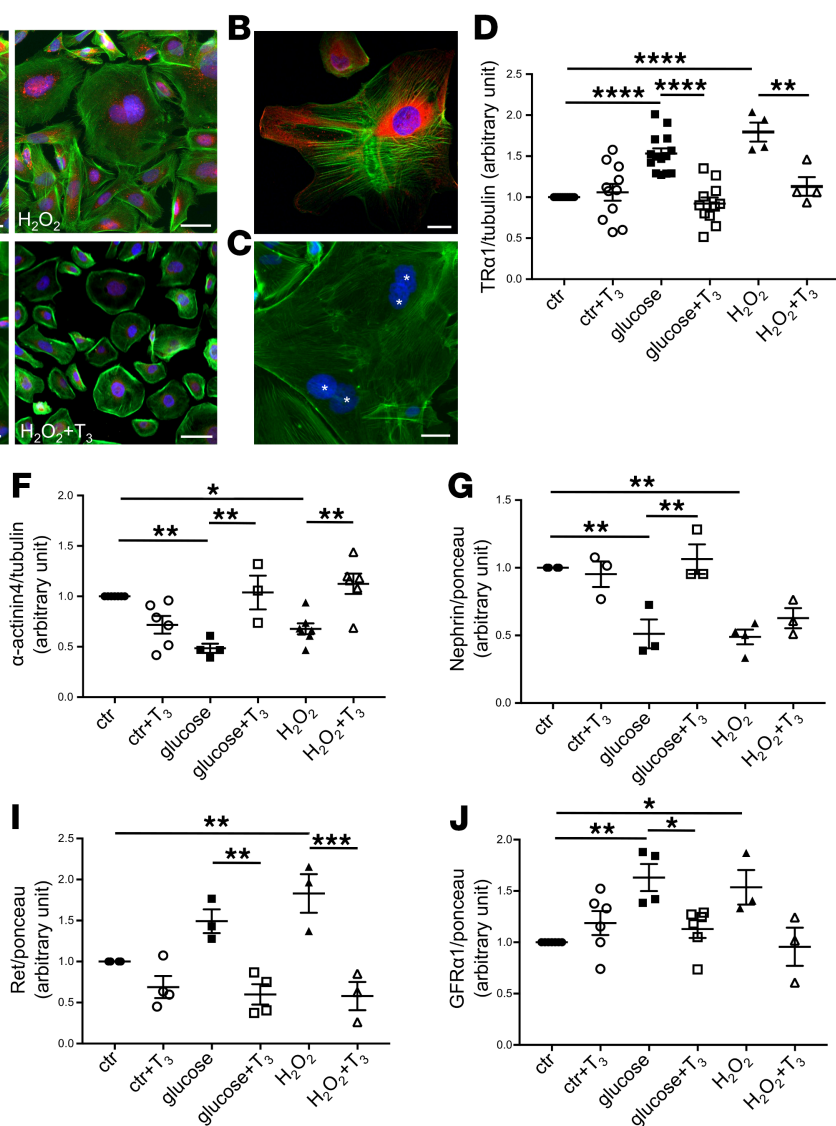

$\mathbf{L}$

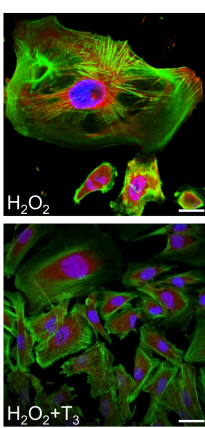

Figure 5. High glucose and oxidative stress induce TR $\alpha 1$ reexpression and podocyte dedifferentiation in vitro that are reversed by $\mathbf{T}_{3^{*}}$ (A) Representative images of $\mathrm{TR} \alpha 1$ (red) and F-actin (green) staining in human podocytes. Both high levels of glucose and $\mathrm{H}_{2} \mathrm{O}_{2}$ induced TR $\alpha 1$ overexpression and partial translocation from the nucleus to cytoplasm and increased cell size (top). $T_{3}$ treatment of stimulated podocytes decreased TR $\alpha 1$ expression and restored its nuclear and perinuclear localization, and reduced cell size, but had no effect on control cells (bottom). (B) A representative image of TR $\alpha 1$ (red) and F-actin (green) staining of injured podocytes. (C) Multinucleated podocytes were visible after glucose treatment (asterisks). (D) Densitometric analysis of TR $\alpha 1$ protein levels in podocytes by Western blot. Both glucose and $\mathrm{H}_{2} \mathrm{O}_{2}$ increased TR $\alpha 1$ expression significantly, and $\mathrm{T}_{3}$ treatment markedly reversed this effect. (E) Quantification of podocytes with peripheral F-actin distribution. Diabetic stimuli caused $F$-actin rearrangements that were reversed by $T_{3}$. (F-J) Densitometric analysis of (F) $\alpha$-actinin4, (G) nephrin, (H) $\alpha$-SMA, (I) Ret, and (J) GFR $\alpha 1$ proteins by Western blot. Diabetic stimuli decrease the expression of adult podocyte markers (F and $\mathbf{G}$ ), cause the reexpression of the mesenchymal marker $\alpha$-SMA $(\mathbf{H})$, and increase Ret (I), and GFR 1 (J) protein levels. $T_{3}$ treatment markedly reversed all these changes. (K) Representative images of $\mathrm{DIO}$ (red) and F-actin (green) staining in human podocytes. Both high glucose and $\mathrm{H}_{2} \mathrm{O}_{2}$ stimulation induced $\mathrm{DIO}_{3}$ upregulation mostly in the nuclear and perinuclear regions (top). $\mathrm{T}_{3}$ treatment of injured podocytes decreased DIO3 expression while having no effect on control cells (bottom). (L) Densitometric analysis of DIO3 protein levels in podocytes by Western blot. Both glucose and $\mathrm{H}_{2} \mathrm{O}_{2}$ markedly increased $\mathrm{DIO}$ expression compared with control. $T_{3}$ treatment reversed this effect. Data from 3 independent experiments are expressed as mean \pm SEM. ${ }^{*} P<0.05 ;{ }^{* *} P<0.01 ;{ }^{* *} P<0.001 ;{ }^{* * *} P<0.0001$, 1-way ANOVA corrected with Tukey's post hoc test. $n$ = 3-15 samples per each condition. Tubulin protein expression ( $\mathbf{D}$ and $\mathbf{F}$ ) or ponceau red staining $(\mathbf{G}-\mathbf{J}$ and $\mathbf{L}$ ) was used as loading control. Scale bars: $50 \mu \mathrm{m}$. 

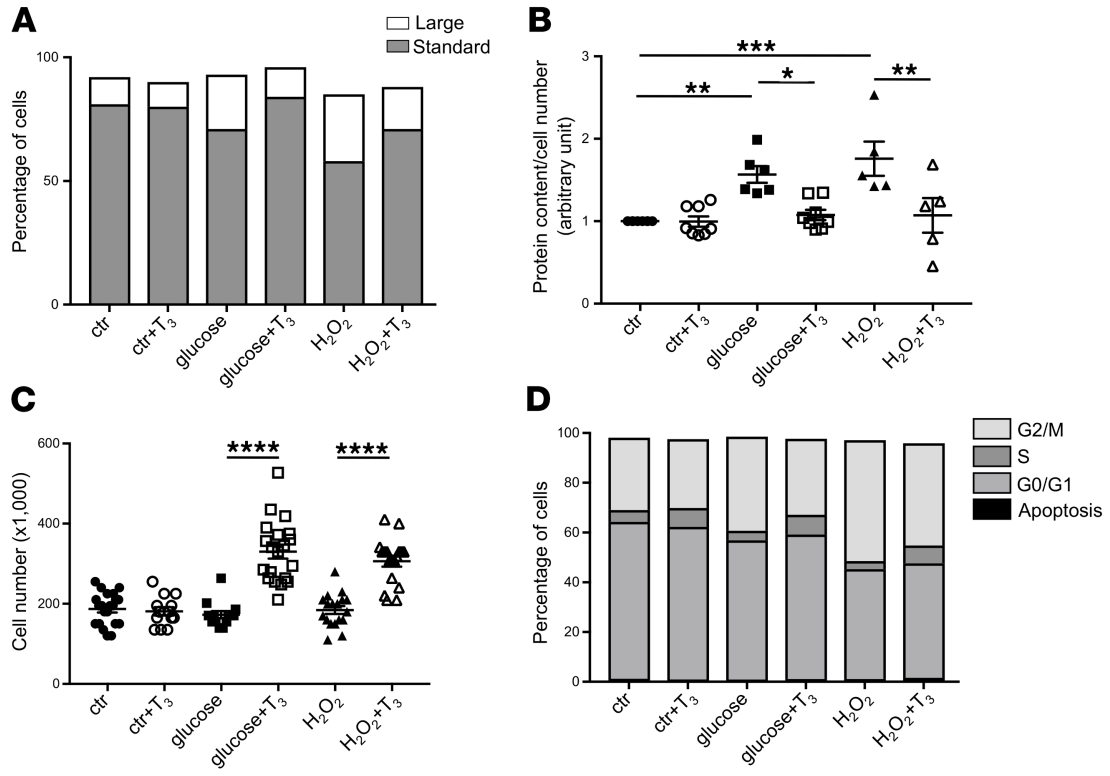

$\mathbf{E}$

$\mathbf{F}$
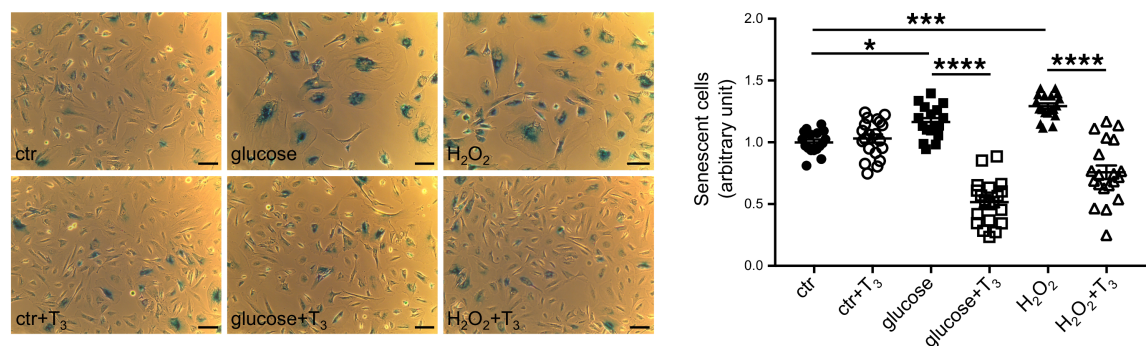

Figure 6. $T_{3}$ administration induces cell proliferation and restores cell size and normal cell cycle distribution in damaged podocytes. (A) Percentage of standard or large treated and untreated podocytes analyzed with FACS by plotting FSC (as dimension of cells) versus SSC (as granularity of cells). Both high levels of glucose and $\mathrm{H}_{2} \mathrm{O}_{2}$ increased the large cell population and $\mathrm{T}_{3}$ restored the standard cell size. (B) Quantification of hypertrophy expressed as total protein content per cell number. Diabetic stimuli induced cell hypertrophy and $\mathrm{T}_{3}$ reversed this effect. (C) Quantification of cell number in treated and untreated podocytes by manual cell counting. $T_{3}$ strongly increased proliferation in podocytes exposed to high levels of glucose or $\mathrm{H}_{2} \mathrm{O}_{2}$. (D) A representative graph of the percentage of podocytes in the cell cycle phases evaluated by FACS. Diabetic stimuli induced a marked $\mathrm{G}_{2} / \mathrm{M}_{\text {accumulation, while }} \mathrm{T}_{3}$ administration reduced $\mathrm{G}_{2} / \mathrm{M}$ phase arrest and increased $\mathrm{S}$ phase cells. $\mathrm{T}_{3}$ treatment had no effect on cell size, hypertrophy, proliferation, and cell cycle distribution in control podocytes. Data from 3 independent experiments are expressed as mean \pm SEM. (E) Representative images of senescence-associated $\beta$-galactosidase (SA- $\beta$-Gal) staining in cultured human podocytes. Scale bar: $100 \mu \mathrm{m}$. (F) Quantification of senescent podocytes expressed as SA- $\beta$-Gal-positive cells per cell number. Both stress stimuli increased the numbers of SA- $\beta$-Cal-positive cells (blue) compared with control, while $\mathrm{T}_{3}$ administration strongly reduced the amount of senescent cells. SA- $\beta$-Gal activity did not differ between control groups. To quantify the number of positive cells, representative images were taken randomly from diverse areas of cell cultures. Number of fields analyzed: $n=17$ for ctr, $n=20$ for ctr $+\mathrm{T}_{3}, n=17$ for glucose, $n=20$ for glucose $+\mathrm{T}_{3}, n=19$ for $\mathrm{H}_{2} \mathrm{O}_{2}, n=$ 20 for $\mathrm{H}_{2} \mathrm{O}_{2}+\mathrm{T}_{3}$. Data are expressed as mean $\pm \mathrm{SEM}$. ${ }^{*} P<0.05 ;{ }^{* *} P<0.01 ;{ }^{* * *} P<0.001 ;{ }^{* * *} P<0.0001,1$-way ANOVA with Tukey's post hoc test.

$0.68 \pm 0.05, P<0.01$ ) and nephrin (glucose $+\mathrm{T}_{3}, 1.06 \pm 0.11$ vs. glucose, $0.51 \pm 0.11, P<0.01 ; \mathrm{H}_{2} \mathrm{O}_{2}+$ $\mathrm{T}_{3}, 0.63 \pm 0.07$ vs. $\mathrm{H}_{2} \mathrm{O}_{2}, 0.49 \pm 0.05$ ) to control levels (Figure 5, F and G); (b) reversed the diabetic stimuli-induced upregulation of the mesenchymal marker $\alpha$-SMA (glucose $+\mathrm{T}_{3}, 0.97 \pm 0.06$ vs. glucose, $1.46 \pm$ $0.11, P<0.001 ; \mathrm{H}_{2} \mathrm{O}_{2}+\mathrm{T}_{3}, 0.73 \pm 0.05$ vs. $\mathrm{H}_{2} \mathrm{O}_{2}, 1.33 \pm 0.06, P<0.0001$ ) (Figure $5 \mathrm{H}$ ); and (c) normalized the high glucose- and $\mathrm{H}_{2} \mathrm{O}_{2}$-induced upregulation of Ret (glucose $+\mathrm{T}_{3}, 0.60 \pm 0.13$ vs. glucose, $1.49 \pm 0.14$, $P<0.01 ; \mathrm{H}_{2} \mathrm{O}_{2}+\mathrm{T}_{3}, 0.58 \pm 0.17$ vs. $\mathrm{H}_{2} \mathrm{O}_{2}, 1.83 \pm 0.24, P<0.001$ ) and GFR $\alpha 1$ (glucose $+\mathrm{T}_{3}, 1.13 \pm 0.09$ vs. glucose, $1.63 \pm 0.13, P<0.05 ; \mathrm{H}_{2} \mathrm{O}_{2}+\mathrm{T}_{3}, 0.96 \pm 0.18$ vs. $\mathrm{H}_{2} \mathrm{O}_{2}, 1.54 \pm 0.17$ ) (Figure 5, I and J) that we also observed in vivo in diabetic rats (Figure $3, \mathrm{E}$ and $\mathrm{F}$ ). $\mathrm{T}_{3}$ treatment also restored the normal DIO3 expression pattern in injured podocytes (glucose $+\mathrm{T}_{3}, 1.10 \pm 0.09$ vs. glucose, $1.49 \pm 0.12, P<0.05 ; \mathrm{H}_{2} \mathrm{O}_{2}+\mathrm{T}_{3}, 0.99$ \pm 0.08 vs. $\mathrm{H}_{2} \mathrm{O}_{2}, 1.31 \pm 0.07, P<0.05$ ) (Figure $5, \mathrm{~K}$ and L). 
Together these data indicate that high glucose and oxidative stress induced the overexpression of $\mathrm{TR} \alpha 1$ and, concurrently, podocyte dedifferentiation and reactivation of fetal genes, structural remodeling, and cell growth. The ability of $\mathrm{T}_{3}$ to normalize TR $\alpha 1$ and DIO3 expression and totally reverse injury-induced phenotypical and molecular changes demonstrates that the TH-TR $\alpha 1$ axis is crucially involved in $\mathrm{DN}$-associated podocyte damage.

$T_{3}$ administration enables the cytokinesis in diabetic stress-damaged podocytes in vitro. To study in depth the role of TH signaling in changes in podocyte size, we performed FACS analysis of untreated and treated human podocytes. Using forward-scattered light (FSC) versus side-scattered light (SSC) scatter plots, we obtained 2 podocyte subpopulations: cells of normal size that we named "standard cells," and cells of increased dimensions that we called "large cells." Scatter plot analysis showed that exposure to high glucose increased the percentage of large cells markedly (glucose, $27.50 \% \pm 2.06 \%$ vs. untreated cells, $15.93 \% \pm 1.98 \%, P<0.01$ ) and, as a consequence, decreased the percentage of standard cells (glucose, $69.47 \% \pm 1.74 \%$ vs. untreated cells, $81.50 \% \pm 1.36 \%, P<0.05$ ) (Figure 6A). Similar effects were observed in $\mathrm{H}_{2} \mathrm{O}_{2}$-stimulated podocytes (large cells, $30.03 \% \pm 2.19 \%$ vs. untreated cells, $P<0.001$; standard cells, $58.00 \% \pm 0.58 \%$ vs. untreated cells, $P<0.0001$ ) (Figure $6 \mathrm{~A}$ ). Treating control podocytes with $\mathrm{T}_{3}$ did not alter cell distribution compared with untreated cells (large cells, $15.25 \% \pm 2.78 \%$; standard cells, $80.23 \% \pm$ $2.04 \%$ ), while treating glucose- or $\mathrm{H}_{2} \mathrm{O}_{2}$-stimulated podocytes with $\mathrm{T}_{3}$ significantly decreased the fraction of large cells (glucose $+\mathrm{T}_{3}, 14.28 \% \pm 0.99 \%$ vs. glucose, $P<0.01 ; \mathrm{H}_{2} \mathrm{O}_{2}+\mathrm{T}_{3}, 17.48 \% \pm 0.51 \%$ vs. $\mathrm{H}_{2} \mathrm{O}_{2}, P<$ 0.01 ) and significantly increased the fraction of standard cells (glucose $+\mathrm{T}_{3}, 82.07 \% \pm 1.53 \%$ vs. glucose, $P$ $<0.01 ; \mathrm{H}_{2} \mathrm{O}_{2}+\mathrm{T}_{3}, 76.53 \% \pm 3.65 \%$ vs. $\mathrm{H}_{2} \mathrm{O}_{2}, P<0.001$ ) (Figure $6 \mathrm{~A}$ and Supplemental Figure $3, \mathrm{~A}$ and $\mathrm{B}$ ).

We then measured the total protein content/cell number to evaluate the effect of $\mathrm{T}_{3}$ treatment on cell hypertrophy. Both high glucose and $\mathrm{H}_{2} \mathrm{O}_{2}$ caused a significant increase in podocyte hypertrophy (1.57 \pm 0.10 -fold and $1.76 \pm 0.21$-fold vs. control, respectively), and this effect was reversed by $\mathrm{T}_{3}$ treatment (glucose $+\mathrm{T}_{3}, 1.08 \pm 0.06$-fold and $\mathrm{H}_{2} \mathrm{O}_{2}+\mathrm{T}_{3}, 1.07 \pm 0.21$-fold vs. control) (Figure 6B). Interestingly, $\mathrm{T}_{3}$ also had a significant proliferative effect in glucose- and $\mathrm{H}_{2} \mathrm{O}_{2}$-stimulated podocytes, while it did not alter cell number of control podocytes (Figure 6C).

We used FACS analysis to further evaluate the proliferative effect of $\mathrm{T}_{3}$ and the possible changes in cell cycle distribution in untreated and treated human podocytes. Exposure to high glucose caused a persistent $\mathrm{G}_{2} / \mathrm{M}$ accumulation $(1.56 \pm 0.11$-fold vs. control, $P<0.001)$ that was similar to that observed in $\mathrm{H}_{2} \mathrm{O}_{2}$-stimulated podocytes $(1.81 \pm 0.14$-fold vs. control, $P<0.0001)$, indicating hypertrophic podocytes inability to conclude cytokinesis. Treating glucose- or $\mathrm{H}_{2} \mathrm{O}_{2}$-stimulated podocytes with $\mathrm{T}_{3}$ decreased the $\mathrm{G}_{2} / \mathrm{M}$ phase arrest (glucose $+\mathrm{T}_{3}, 1.01 \pm 0.04$ vs. glucose, $1.56 \pm 0.11, P<0.01 ; \mathrm{H}_{2} \mathrm{O}_{2}+\mathrm{T}_{3}, 1.27 \pm 0.08$ vs. $\mathrm{H}_{2} \mathrm{O}_{2}, 1.81 \pm 0.14, P<0.01$ ) and increased $\mathrm{S}$ phase cells markedly (glucose $+\mathrm{T}_{3}, 1.98 \pm 0.34$ vs. glucose, $0.97 \pm 0.07, P<0.001 ; \mathrm{H}_{2} \mathrm{O}_{2}+\mathrm{T}_{3}, 1.28$ \pm 0.07 vs. $\mathrm{H}_{2} \mathrm{O}_{2}, 0.78 \pm 0.04$ ) (Figure $6 \mathrm{D}$ and Supplemental Figure $3 \mathrm{D}$ ). According to the cell number quantification (Figure $6 \mathrm{C}$ ), $\mathrm{T}_{3}$ treatment did not induce any changes in $\mathrm{G}_{2} / \mathrm{M}$ and $\mathrm{S}$ phase in control podocytes $\left(\mathrm{G}_{2} / \mathrm{M}\right.$ : control $+\mathrm{T}_{3}, 1.05 \pm 0.03$-fold vs. control; $\mathrm{S}$ : control $+\mathrm{T}_{3}, 1.08 \pm 0.13$-fold vs. control) (Supplemental Figure 3 , $\mathrm{C}$ and $\mathrm{D})$. Together, these data clearly demonstrate that high glucose and oxidative injury induced podocytes to reenter the cell cycle, where they arrested in the $\mathrm{G}_{2} / \mathrm{M}$ phase undergoing hypertrophy; in these podocytes, $\mathrm{T}_{3}$ enabled cytokinesis, which translated into hypertrophy reduction and cell number increase (Figure 6, B and C). Since diabetes-induced cell cycle arrest is linked to premature senescence $(37,38)$, we tested whether TH signaling plays a role in this process. Both high glucose and $\mathrm{H}_{2} \mathrm{O}_{2}$ caused a significant increase in the number of senescence-associated $\beta$-galactosidase-positive (SA- $\beta$-Gal-positive) podocytes (39), and this effect was again reversed by $\mathrm{T}_{3}$ treatment (Figure 6, $\mathrm{E}$ and $\mathrm{F}$ ), indicating that $\mathrm{TH}$ signaling plays an important role in diabetes-associated podocyte senescence and that its activation results in strong antiaging effects.

TRa1 induces hypertrophy through the ERK1/2 pathway. To identify the underlying mechanisms of action of TR $\alpha 1$, we analyzed changes in ERK1/2 signaling pathways, which are regulated by the TH-TR axis and involved in hypertrophy, dedifferentiation, proliferation, and apoptosis (40). Neither high glucose nor $\mathrm{T}_{3}$ treatment affected basal ERK1/2 expression (control $+\mathrm{T}_{3}, 1.32 \pm 0.21$; glucose, $1.31 \pm 0.18$; and glucose + $\mathrm{T}_{3}, 1.15 \pm 0.36$-fold vs. control) (Figure 7A), while high glucose significantly increased phospho-ERK1/2 (pERK1/2) $(1.57 \pm 0.10$ vs. control, $P<0.05)$ that was restored to control levels through $\mathrm{T}_{3}$ treatment (glucose $+\mathrm{T}_{3}, 1.03 \pm 0.11$ vs. glucose, $P<0.05$ ) (Figure 7B). The activation of ERK1/2 after glucose stimulation and its modulation by $\mathrm{T}_{3}$ treatment suggested that ERK1/2 plays a role as a TR $\alpha 1$ mediator for cell hypertrophy in podocytes in vitro. To confirm our hypothesis, we first tested whether this glucose-induced alteration was indeed mediated by ERK1/2 by treating podocytes with a subtoxic concentration of UO126 
A
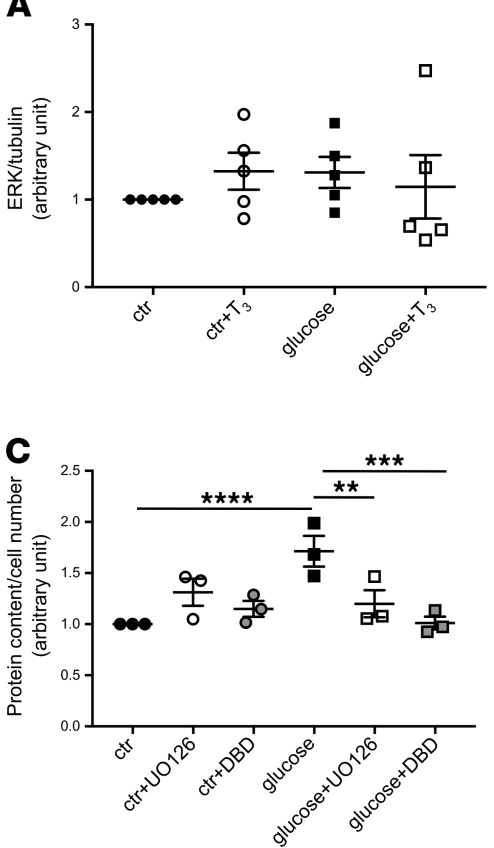

B
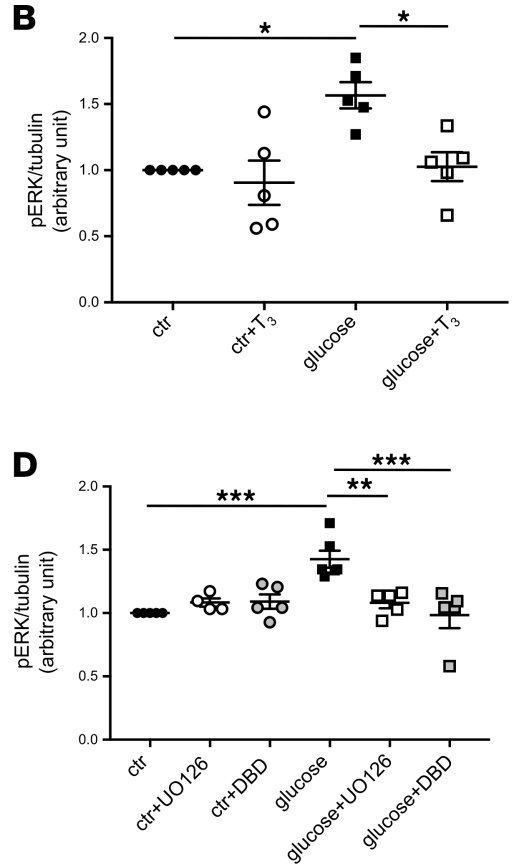

Figure 7. TR $\alpha 1$ regulates glucose-induced hypertrophy through downstream activation of ERIK1/2. (A and B) Densitometric analysis of (A) ERK1/2 and (B) pERK1/2 protein in podocytes by Western blot. (A) Podocyte total ERK1/2 protein expression was not changed either by glucose or $\mathrm{T}_{3}$ treatment. (B) Glucose induced an increase in pERK1/2 protein levels, which were significantly reduced by $T_{3}$ treatment. (C) Quantification of hypertrophy expressed as protein content per cell number. Glucose induced podocyte hypertrophy, which was prevented by U0126 and DBD. Neither U0126 nor DBD had any effect on untreated cells. (D) Densitometric analysis of pERK1/2 protein in podocytes by Western blot. The increase in pERK1/2 protein levels in glucose-stimulated podocytes was prevented by administering U0126 or DBD. Tubulin protein expression was used as sample loading control (A, B, and D). Data from 3 independent experiments are expressed as mean \pm SEM. ${ }^{*} P<0.05$; ${ }^{* *} P<0.01$; ${ }^{* * *} P<0.001{ }^{* * * *} P<0.0001,1$-way ANOVA with Tukey's post hoc test. $n=3-6$. pERK1/2, phospho-ERK1/2.

— an inhibitor of ERK1/2 phosphorylation - alone or in combination with high glucose for 9 days. We observed that UO126 treatment impeded the establishment of hypertrophy induced by glucose alone (glucose + UO126, $1.20 \pm 0.13$ vs. glucose, $1.71 \pm 0.15, P<0.01$ ) (Figure 7C), demonstrating that ERK1/2 is a mediator of hypertrophy in glucose-stimulated podocytes. Second, to confirm that TR $\alpha 1$ plays a role in regulating hypertrophy in glucose-stimulated cells, we treated podocytes with debutyl-dronedarone (DBD) - a TR $\alpha 1$ inhibitor (41) - alone or in combination with high glucose for 9 days. Treatment with DBD, in combination with glucose, blocked the hypertrophy that was induced by glucose alone (glucose $+\mathrm{DBD}$, $1.01 \pm 0.06$ vs. glucose, $1.71 \pm 0.15, P<0.001$ ) (Figure $7 \mathrm{C}$ ), demonstrating that TR $\alpha 1$ is necessary for hypertrophy induction. Finally, to assess whether ERK1/2 acts downstream in the TR 1 pathway, we evaluated the expression of pERK1/2 after DBD treatment (Figure 7D). Remarkably, treatment with DBD prevented ERK1/2 activation (glucose $+\mathrm{DBD}, 0.98 \pm 0.10$ vs. glucose, $1.43 \pm 0.07, P<0.001$ ) as much as UO126 treatment did (glucose + UO126, $1.08 \pm 0.04$ vs. glucose, $P<0.01$ ) (Figure 7D). Neither UO126 nor DBD affected pERK1/2 levels in control podocytes (Figure 7D).

These findings indicate that the TR $\alpha 1$ is responsible for hypertrophy induction and its action is mediated downstream by the ERK1/2 pathway (Figure 8 ).

\section{Discussion}

Low systemic $\mathrm{T}_{3}$ level is the most frequent alteration of the $\mathrm{TH}$ signaling profile that is observed in chronically ill patients (42). This alteration has long been considered as a harmless metabolic adaptation to chronic illness. However, recent evidence shows that alterations of the TH signaling profile associate with radical phenotypical changes in terminally differentiated cells of various types (i.e., cardiomyocytes [ref. 43], pancreatic cells [ref. 44], neuronal cells [ref. 45], and skeletal muscle cells [ref. 46]). Both the changes in $\mathrm{T}_{3}$ availability and in podocytes' phenotype have been already reported in DN; however, whether these phenomena are interconnected or not is still unknown. 
Adult TH/TRa1 axis pattern:

- Normal $T_{3}$ levels

- TRa1 acts as holoreceptor

(liganded state)

Fetal TH/TR $\alpha 1$ axis pattern:

$\downarrow T_{3}$ levels

† TR $\alpha 1$ acts as aporeceptor (unliganded state)
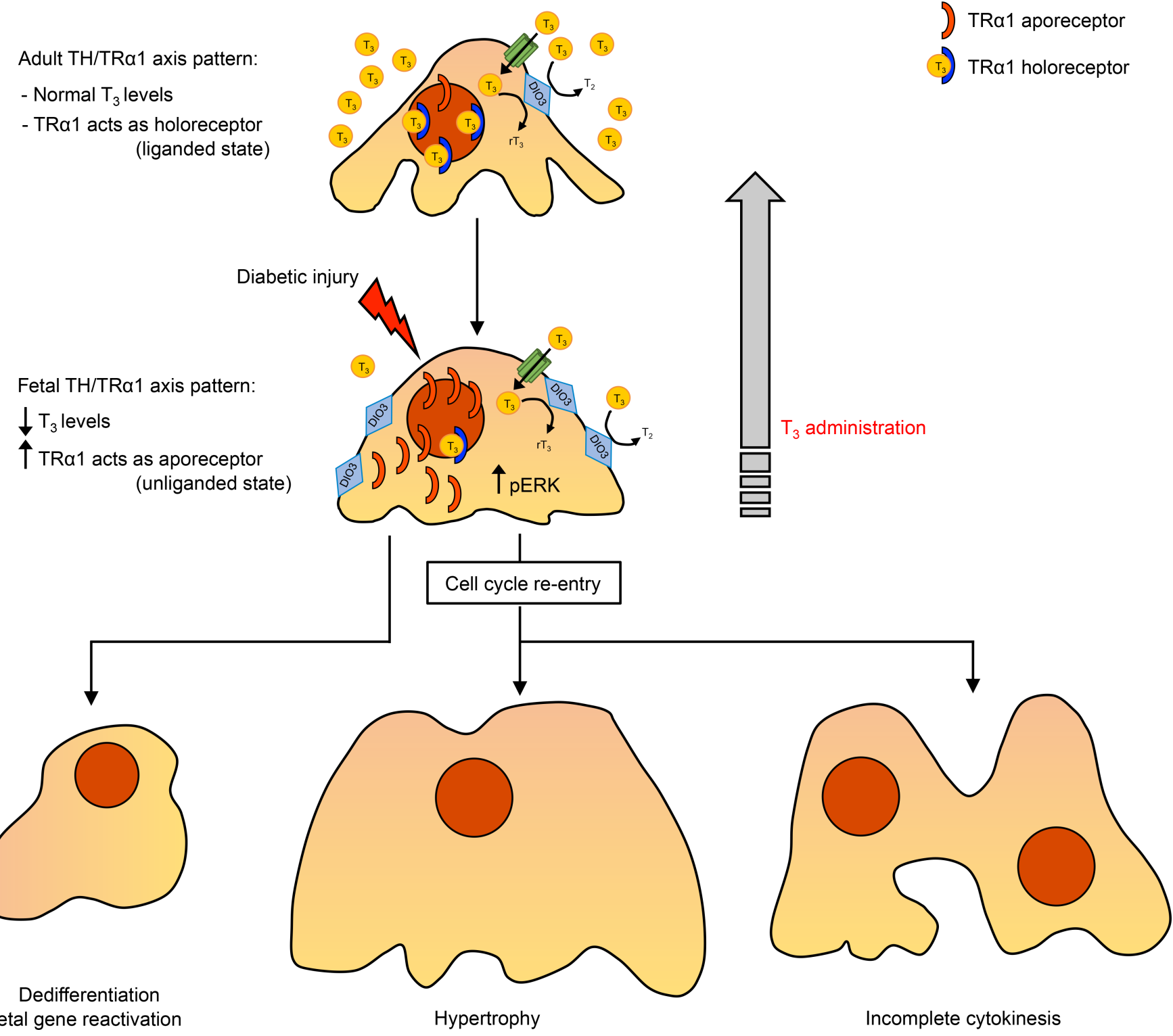
Fetal gene reactivation

Hypertrophy

ncomplete cytokinesis

Figure 8. TH/TR $\alpha 1$ axis actions in diabetes. In healthy podocytes, $T_{3}$ levels are maintained within a physiological range and TR 1 acts as holoreceptor. Local $T_{3}$ availability is controlled by the $T_{3}$-inactivating enzyme DIO3, which converts excessive $T_{3}$ into $\mathrm{rT}_{3}$ and $\mathrm{T}_{2}$. In response to diabetic injury, systemic $\mathrm{T}_{3}$ levels drop markedly, and TR $\alpha 1$ and $\mathrm{DIO}$ are overexpressed locally, resulting in the coordinated adoption of the fetal ligand/receptor relationship profile (i.e., low $T_{3}$ availability/high local TR $\alpha 1$ ). Here, TR $\alpha 1$ acts as aporeceptor (as happens in the fetus), repressing adult genes, triggering the reactivation of the podocyte developmental program, activating ERK1/2, and inducing cells to reenter the cell cycle. Unable to complete cytokinesis, mitotically activated podocytes become hypertrophic and polynucleated. $T_{3}$ treatment switches TR $\alpha 1$ to the holoreceptor state (as happens after birth), promoting a mature cell phenotype and globally reversing the phenotypical alterations induced by diabetes. $\mathrm{rT}_{3}$, reverse L-triiodothyronine; $\mathrm{T}_{2}$, $\mathrm{L}$-diiodothyronine.

This study is the first to our knowledge to demonstrate that the TH-TR axis adopts a fetal relationship profile in kidney in response to diabetic stress that may play a pivotal role in the diabetes-induced pathological growth, remodeling, and dedifferentiation of podocytes and identifies TR $\alpha 1$ as a key regulator of the pathogenesis of DN.

By analyzing ZSF1 diabetic rats at different stages of DN, we observed a progressive decrease in $\mathrm{T}_{3}$ availability that paralleled the worsening of metabolic disease, the increase of proteinuria, and the onset of glomerular structural alterations. The reduction of $\mathrm{T}_{3}$ levels was accompanied by the reexpression of the predominantly fetal TR isoform TR $\alpha 1$ in podocytes and parietal cells of diabetic rats, and similar expression patterns were observed in patients with DN. Moreover, the expression of the TH-inactivating enzyme DIO3 also increased at the glomerular level in ZSF1 diabetic rats. TR $\alpha 1$ is highly expressed during fetal life, when $\mathrm{T}_{3}$ levels are low, and acts as aporeceptor (unliganded state) to repress adult genes. After birth, a significant 
increase in $\mathrm{T}_{3}$ levels occurs and $\mathrm{TR} \alpha 1$ switches to holoreceptor (liganded state) to induce the expression of adult genes and promote cell maturation and physiological growth. Local $\mathrm{T}_{3}$ availability is controlled by DIO3 in a spatiotemporal manner during organ development and maturation. Specifically, DIO3 is highly expressed in fetal tissues to inactivate intracellular $\mathrm{T}_{3}$ and enable cell proliferation and physiological growth. Around birth, DIO3 expression is turned off in virtually every tissue to enable the local increase in $\mathrm{T}_{3}$ and subsequent cell differentiation and tissue maturation (47); these changes drive the fetal-to-adult switch in gene expression. Robust local reexpression of DIO3 has been observed in adulthood in fish epimorphic regeneration (48) and in mammals in conditions under which low $\mathrm{TH}$ is necessary to sustain cell proliferation and growth, as in inflammation $(49,50)$, tumors (51), and liver regeneration (52) as well as in the surviving cardiomyocytes after myocardial infarction (53). Here, we report that DIO3 also increases locally in the diabetic kidney and that this is associated with low $\mathrm{T}_{3}$ levels and the overexpression of TR $\alpha 1$. Together, these findings indicate that TH signaling coordinately adopts a fetal ligand/receptor relationship profile (i.e., low $\mathrm{T}_{3}$ availability/high local TR $\alpha 1$ and high DIO3) that may be responsible for the fetal/dedifferentiation features observed in diabetic podocytes. This hypothesis is strengthened by the fact that, in ZSF1 rats, TR $\alpha 1$ increased massively in cells that also expressed fetal kidney markers as well as in dedifferentiating desmin-positive podocytes. To dissect the biological significance of the recapitulation of the fetal profile of TH signaling and its link with diabetic podocyte alterations, we performed mechanistic studies in vitro. In podocytes treated with high glucose or $\mathrm{H}_{2} \mathrm{O}_{2}$, we observed a strong increase of TR $\alpha 1$ expression that was associated with changes in the actin-based cytoskeleton, downregulation of adult podocyte markers, upregulation of mesenchymal markers, and increased expression of Ret, GFR $\alpha 1$, and DIO3. Moreover, high glucose and oxidative injury induced podocytes to reenter the cell cycle, which they were unable to conclude, and remained blocked in the $\mathrm{G}_{2} / \mathrm{M}$ phase undergoing hypertrophy. Strikingly, $\mathrm{T}_{3}$ treatment globally reversed the changes observed in stimulated podocytes and enabled the completion of cytokinesis, demonstrating that TH signaling is a key player in the phenotypical and molecular changes that occur in podocytes in response to exposure to the typical components of the diabetic milieu. It is important to highlight that in the absence of any injury $\mathrm{T}_{3}$ did not affect cell cycle or growth, suggesting that the $\mathrm{T}_{3}$-mediated proliferative effect occurs exclusively in damaged podocytes. Finally, to investigate the pathways underlying the effects of TR $\alpha 1$ overexpression, we performed pharmacological studies with specific inhibitors and identified ERK $1 / 2$ as the downstream mediator of the TH-TR $\alpha 1$ axis in inducing podocyte hypertrophy.

TR $\alpha 1$ acting as a molecular switch that triggers the recapitulation of the fetal cell phenotype in response to injury in terminally differentiated and highly specialized cells has also been observed in cardiomyocytes. Stress stimuli, such as adrenergic stimulation induced the upregulation of TR $\alpha 1$ in isolated cardiomyocytes (54), reexpression of fetal structural proteins, and the adoption of fetal-like morphology in terms of cellular structure, size, shape, and geometry (55). As we observed in diabetic podocytes, stress-induced morphological and molecular alterations of the cardiomyocytes were mediated by ERK1/2, and $\mathrm{T}_{3}$ treatment reduced TR $\alpha 1$ expression, reversed the fetal-like pattern of contractile protein expression, both in vitro and in vivo, and normalized wall tension and cardiac chamber geometry in experimental heart disease (56). Of great interest, changes in the TH-TR $\alpha 1$ axis have also been observed in the diabetic heart (57-59), the ischemic brain (60), and injured skeletal muscle (61). These observations, in combination with our data, suggest that the TH-TR $\alpha 1$ axis is a highly sensitive pleiotropic pathway that is able to respond to a wide range of different noxious stimuli (including hemodynamic, ischemic and adrenergic, high glucose, and oxidative stress) and induce profound phenotypical changes in adult injured tissues. The significant differences between hypothyroidism- and diabetes-induced phenotypical changes (atrophy vs. hypertrophy; unchanged podocyte number vs. podocyte loss, respectively) $(62,63)$ indicate that the low availability of T3 alone does not suffice to trigger the recapitulation of the fetal podocyte phenotype and remodeling; one or more chronic noxious stimuli are required to induce TH signaling to adopt its fetal ligand/receptor relationship profile (i.e., low T3 availability/high local TR $\alpha$ ), which in turn triggers, and possibly coordinates, the dedifferentiation in diabetic podocytes.

One compelling question remains unanswered: why does the fetal $\mathrm{TH}$ signaling profile recur in diabetic podocytes? It could be either an adaptation to chronic illness (e.g., inducing a low energy state and/or compensatory growth) or a maladaptation (e.g., inducing phenotypical changes that are more harmful than beneficial). It is possible that low $\mathrm{T}_{3}$ indeed provides an initial metabolic benefit to the damaged cells and possibly allows the cells to grow in size to compensate increased work overload. However, persistent low availability of $\mathrm{T}_{3}$ induces also the changes in $\mathrm{TH}$ signaling that trigger the dedifferentiation and reactivation of downstream developmental pathways. In any case, based on the ubiquitous roles of $\mathrm{TH}$ signaling in the 
development of all vertebrates and epimorphic regeneration in various taxa $(48,64)$, we can hypothesize that the fetal relationship pattern being readopted (low $\mathrm{T}_{3} /$ high $\mathrm{TR} \alpha 1$ ) during chronic stress is an evolutionary remnant of TH-TR axis-mediated tissue regeneration (2). Though capable of initiating regeneration through developmental pathways, it fails to conclude the regeneration process because this ability has been lost during evolution with the increased complexity of mammalian organs. Hence, the phenotypical and morphological alterations in terminally differentiated podocytes in response to diabetic injury may be manifestations of an imperfect/maladaptive recapitulation of early developmental steps mediated by the TH/ TR $\alpha 1$ axis (Figure 8). However, even if it is a maladaptive activation of developmental pathways, it can also be used as a window for regeneration: controlling these pathways spatiotemporally, it could be a strategy to direct the regeneration of damaged tissues. In light of the results reported here, one could propose that pharmacological modulation of the TH-TR axis through $\mathrm{T}_{3}$ treatment could potentially be a promising therapeutic strategy for reversing diabetes-induced pathological podocyte responses and hopefully to increase the regeneration potential of diabetic kidneys. Nevertheless, translating this strategy into clinical practice will not be easy. To achieve an efficient therapeutic effect on stressed cells (overexpressing both TR $\alpha 1$ and DIO3) under conditions of systemic hypothyroidism, very high doses of $\mathrm{T}_{3}$ would be needed; but this can cause various adverse effects - such as tachycardia, arrhythmias (65), hyperfiltration (66), dysfunction of the thyroid gland (67), and even death in the case of long-term treatment (68). As a matter of fact, when we tried to test the therapeutic potential of $\mathrm{T}_{3}$ treatment in diabetic rats (our unpublished data), we needed to use very high doses of $\mathrm{T}_{3}$ in order to reach an initial beneficial effect in function; however, this caused significant complications and we needed to interrupt the study. To minimize the adverse effects and maximize the reparative and regenerative capacities of $\mathrm{TH}$, (our) future therapeutic approaches should be focused on developing a drug delivery technology that could (a) mainly (if not exclusively) target the stressed cells and (b) carry and deliver the TH into the cells of interest.

Although important research remains to be done to shed light on the role that TH signaling plays in the pathobiology of diabetic kidney, we can say that our data provide what we believe to be a novel perspective on the pathogenesis of $\mathrm{DN}$ and may provide a conceptual basis for developing potentially new regenerative therapeutic approaches.

\section{Methods}

Animals. Male ZSF1 (ZSF1-Lepr ${ }^{\mathrm{fa}}$ Lepr $^{\mathrm{cp}} / \mathrm{Crl}$ ) and aged-matched nondiabetic lean rats (ZSF1-Lepr ${ }^{\mathrm{fa}}$ Lepr $^{\mathrm{cp}} /+$ ) were obtained by Charles River Laboratories Italia. Animals were maintained in a specific pathogen-free facility and kept on a 12:12-hour-light/dark cycle with free access to water. ZSF1 rats were fed with the animal Purina 5008 diet (Charles River) containing $18 \mathrm{~kJ} \%$ fat to accelerate the onset of diabetes. ZSF1 rats at 10 weeks of age underwent unilateral nephrectomy to accelerate the onset of renal disease. All animals were sacrificed at 6 months of age.

Sprague-Dawley (SD) rats (Crl:SD, Charles River Laboratories Italia) were used to study the TR $\alpha 1$ expression pattern in embryonic, postnatal, and adult kidneys. Matings for obtaining SD offspring were performed at Istituto di Ricerche Farmacologiche Mario Negri IRCCS. E13.5 $(n=20)$, E15.5 $(n=16)$, $\mathrm{E} 18.5(n=12), \mathrm{P} 4(n=3), \mathrm{P} 7(n=3)$, and adult $(n=3)$ kidneys were isolated from SD rats.

Tissues from BTBR wild-type and BTBR Lep ${ }^{o b / o b}$ mice at 14 weeks of age, C57BL/6 wild-type mice injected intraperitoneally with saline or streptozotocin (200 mg/ $\mathrm{kg}$ body weight, MilliporeSigma) as previously described (69), and 6-month-old Zucker diabetic fatty rats (ZDF/Gmi-fa/fa) and age-matched nondiabetic lean rats (ZDF/Gmi-fa/+) were used for immunohistochemical studies.

Sera from another set of $\mathrm{ZDF}$ and lean rats at 7 months of age were used for the determination of total $\mathrm{T}_{3}$ levels, as described below.

Urinary protein excretion measurements. Urinary excretion of proteins was measured in 24-hour urine samples by the Coomassie method with a Cobas Mira autoanalyzer (Roche Diagnostics Systems).

$T_{3}$ measurements. Serum total $\mathrm{T}_{3}$ levels $\left(\mathrm{T}_{3}\right.$, ng/dl) were measured using the tT3 total Triiodothyronine ELISA kit (catalog 125-300, Accubind Elisa Microwells, Monobind Inc.) following the manufacturer's instructions. For $\mathrm{T}_{3}$ determination, serum from $n=3-9$ lean rats and $n=5-10$ ZSF1 rats and from $n=10$ $\mathrm{ZDF}$ lean rats and $n=8 \mathrm{ZDF}$ rats was used.

Renal histology. Kidney samples were fixed in Duboscq-Brazil (catalog P0094, Diapath), dehydrated, and embedded in paraffin. Three-micrometer sections were stained with periodic acid-Schiff reagent, and at least 100 glomeruli were examined for each animal. The degree of glomerular damage was assessed by a 
semiquantitative scoring method (index) based on the number of glomeruli with sclerosis: grade 0 , normal glomeruli; grade 1 , up to $25 \%$ involvement; grade 2 , up to $50 \%$ involvement; grade 3 , up to $75 \%$; and grade 4 , greater than $75 \%$ sclerosis. The index was calculated as follows: $[(1 \times \mathrm{N} 1)+(2 \times \mathrm{N} 2)+(3 \times \mathrm{N} 3)+(4 \times$ $\mathrm{N} 4)] /(\mathrm{N} 0+\mathrm{N} 1+\mathrm{N} 2+\mathrm{N} 3+\mathrm{N} 4)$, where $\mathrm{N} x$ is the number of glomeruli in each grade of glomerulosclerosis. Kidney biopsies were analyzed by the same pathologist, who was unaware of the nature of the experimental groups. Samples were examined using ApoTome Axio Imager Z2 (Zeiss).

Immunofluorescence and immunohistochemical analyses. Immunostaining of TR $\alpha 1$ and TR $\beta 1$ was performed in rat and human biopsies. After antigen retrieval, OCT frozen kidney sections were blocked in $1 \%$ BSA and incubated overnight at $4^{\circ} \mathrm{C}$ with the following primary antibodies: rabbit anti-TR $\alpha 1$ and rabbit antiTR $\beta 1$ (catalog PA1-211A and catalog PA1-213A, Invitrogen, 1:250), mouse anti-rat nestin (catalog 556309, BD Biosciences, 1:100), mouse anti-human nestin (catalog MAB5326, Millipore, 1:250), rabbit anti-c-Ret (catalog 3223, Cell Signaling, 1:50), rabbit anti-GFR $\alpha 1$ (catalog sc-10716, Santa Cruz Biotechnology, 1:30), mouse anti-Wilms tumor protein-1 (WT-1) (catalog sc-7385, Santa Cruz Biotechnology, 1:30), rabbit antiPax2 (catalog 71-6000, Invitrogen, 1:200), rabbit anti-Six2 (catalog 11562-1-AP Proteintech, 1:400), followed by appropriate Cy3- or FITC-conjugated secondary antibodies (Jackson ImmunoResearch Laboratories). Nuclei and renal structures were stained with DAPI (catalog D9542, MilliporeSigma, $1 \mu \mathrm{g} / \mathrm{ml}$ ) and with DyLight 649-labeled Lens culinaris agglutinin (catalog DL1048, Vector Laboratories, 1:400), respectively. Negative controls were obtained by omitting the primary antibody on adjacent sections. Samples were examined using an inverted confocal laser microscope (Leica TCS SP8, Leica Biosystems).

For immunohistochemical analyses, formalin-fixed, 3- $\mu$ m paraffin-embedded kidney sections were incubated with Peroxidazed 1 (Biocare Medical) to quench endogenous peroxidase, after antigen retrieval in a decloaking chamber with DIVA buffer. After blocking for 30 minutes with Rodent Block R (Biocare Medical), sections were incubated with rabbit anti-DIO3 (catalog NBP1-05767, Novus Biologicals, 1:2000), rabbit anti-TR $\alpha 1$ (catalog PA1-211A, Invitrogen, 1:300), rabbit anti-Pax2 (catalog 71-6000, Invitrogen, 1:300), rabbit anti-Six2 (catalog 11562-1-AP Proteintech, 1:200), rabbit anti-GDNF (catalog sc-328, 1:200), and rabbit anti-desmin (catalog ab15200, Abcam, 1:2000) antibodies, followed by Rabbit on Rodent HRP-Polymer (catalog RMR622G, Bio-Optica) for 20 minutes at room temperature. To study the glomerular podocyte density and individual podocyte volume, sections were incubated with mouse anti-WT-1 (catalog sc-7385, Santa Cruz Biotechnology, 1:30) and mouse anti-Glepp1 (catalog MABS455, Millipore, 1:100) antibodies followed by Mouse on Rat HRP-Polymer (catalog MRT621G, Bio-Optica) for 30 minutes at room temperature. Stainings were visualized using diaminobenzidine (Biocare Medical) substrate solutions. Slides were counterstained with Mayer's hematoxylin (Bio Optica), mounted with Eukitt mounting medium and finally observed using light microscopy (ApoTome, Axio Imager Z2, Zeiss). Negative controls were obtained by omitting the primary antibody on adjacent sections. Stainings of TR $\alpha 1$ and fetal markers (Pax2, Six2, and GDNF), TR $\alpha$, and desmin as well as WT-1 and Glepp1 were performed in serial sections. Desmin staining was quantified by semiquantitative score from 0 to 3 ( 0 , no staining or traces; 1 , staining in $<25 \%$ of the glomerular tuft; 2 , staining affecting $26 \%-50 \% ; 3$, staining in $>50 \%$ ). The average number of podocytes per glomerulus and the glomerular volume were determined through morphometric analysis as previously described (70). For Glepp1-positive area measurement, 15 consecutive glomerular tuft areas were measured, and the percentage of each glomerular tuft that stained positive using Glepp1 immunoperoxidase was measured using ImageJ software (v 1.51, NIH). Globally sclerotic glomeruli were excluded from analysis. The mean Glepp1-positive (podocyte) volume per glomerulus was estimated by multiplying the mean glomerular volume by the mean percentage of Glepp1-positive area. The number of podocytes per glomerular tuft was measured using WT-1 immunoperoxidase staining, as described above. The mean individual podocyte volume was estimated by dividing the mean Glepp1-positive tuft volume by the mean podocyte number per tuft (71).

Cell culture and treatments. Conditionally immortalized human podocytes (provided by P.W. Mathieson and M.A. Saleem, Children's Renal Unit and Academic Renal Unit, University of Bristol, Southmead Hospital, Bristol, United Kingdom) were cultured and differentiated as described previously (72). Briefly, for cell propagation, podocytes were grown at $33^{\circ} \mathrm{C}$ in standard condition $\left(5 \% \mathrm{CO}_{2}, 20 \% \mathrm{O}_{2}\right)$ in RPMI1640 medium (catalog 21875034, Life Technologies Europe BV) supplemented with 10\% FBS (catalog 10270106, Life Technologies), 1\% insulin-transferrin-selenium (catalog 41400045, Life Technologies), and $1 \%$ Penicillin/Streptomycin (catalog 15140122, Life Technologies). Media were changed every 2 days. For cell differentiation, 80\%-confluent cells were harvested using $0.05 \%$ (1') Trypsin-EDTA (catalog 15400054, Thermo Fisher Scientific) and seeded at a density of $5 \times 10^{3}$ cells $/ \mathrm{cm}^{2}$ in 6 - or 12 -well plates coated with 
$0.1 \mathrm{mg} / \mathrm{ml}$ rat-tail type I collagen (catalog 354236, Corning, Corning-Costar). Podocytes were maintained at $37^{\circ} \mathrm{C}$ for 14 days, and media were changed every 2 days. After differentiation, podocytes were starved in culture medium supplemented with $2 \%$ FBS overnight. Cells were then treated with $40 \mathrm{mM}$ glucose (catalog G5146, MilliporeSigma) or $50 \mu \mathrm{M}$ hydrogen peroxide $\left(\mathrm{H}_{2} \mathrm{O}_{2}\right.$; catalog $\mathrm{H} 1009$, MilliporeSigma) for 3 days and with $40 \mathrm{mM}$ glucose or $50 \mu \mathrm{M} \mathrm{H}_{2} \mathrm{O}_{2}$ in combination with $0.1 \mu \mathrm{M} \mathrm{T}_{3}$ (catalog T6397, MilliporeSig$\mathrm{ma}$ ) for the following 6 days. In other experiments, cells were treated with $40 \mathrm{mM}$ glucose alone or in combination with $0.5 \mathrm{M}$ DBD (catalog D288875, Toronto Research Chemicals) or in combination with $0.1 \mu \mathrm{M}$ UO126 (catalog 9903S, Cell Signaling Technology) for 9 days. Glucose was dissolved in culture medium. $\mathrm{H}_{2} \mathrm{O}_{2}$ was diluted in culture medium. $\mathrm{T}_{3}$ was dissolved in $\mathrm{NaOH}, \mathrm{DBD}$ in methanol, and UO126 in DMSO, and they were further diluted in culture medium. $\mathrm{T}_{3}$ concentration in the culture medium was $0.67 \mathrm{nM}$.

Protein extraction and Western blot analysis. Adherent cells were collected and rinsed with cold $1 \times$ PBS (catalog AM9625, Invitrogen). Cells were then lysed in CelLytic M buffer (catalog C2978, MilliporeSigma) supplemented with protease (catalog 11836153001, MilliporeSigma) and phosphatase (catalog 04906837001, MilliporeSigma) inhibitors, following the manufacturer's instructions. Kidney samples were homogenized, and cells were then lysed in CelLytic MT Cell Lysis Reagent (catalog C3228, MilliporeSigma) supplemented with protease and phosphatase inhibitors. Protein concentration was determined by DC protein assay (catalog 5000116, Bio-Rad Laboratories). Whole-cell lysates (30 $\mu \mathrm{g})$ were separated by SDS polyacrylamide gel electrophoresis and transferred onto nitrocellulose blots (catalog GE10600001, MilliporeSigma). After blocking with 5\% (w/v) BSA (catalog A7906, MilliporeSigma) in Tris buffered saline- $0.1 \%$ (TBS-0.1\%) Tween-20 (catalog P2287, MilliporeSigma), blots were incubated overnight at $4^{\circ} \mathrm{C}$ with the following primary antibodies: rabbit anti-TR $\alpha 1$ (catalog PA1-211A, Invitrogen, 1:500), rabbit anti-Pax2 (catalog 71-600, Invitrogen, 1:400), rabbit anti-Six2 (catalog 11562-1-AP, Proteintech, 1:500), mouse anti-GDNF (catalog sc-13147, Santa Cruz Biotechnology, 1:250), rabbit anti-Ret (catalog ANT-025, Alomone Labs, 1:500), rabbit anti-GFR $\alpha 1$ (catalog sc-10716, Santa Cruz Biotechnology, 1:200), rabbit anti-DIO3 (catalog NBP1-05767, Novus Biologicals, 1:2000), goat anti- $\alpha$-actinin4 (catalog sc-49333, Santa Cruz Biotechnology, 1:200), mouse anti- $\alpha$-SMA (catalog A2547, MilliporeSigma, 1:500), rabbit anti-pERK1/2 (catalog 9101, Cell Signaling, 1:1000), rabbit anti-ERK1/2 (catalog 4695, Cell Signaling, 1:1000), and mouse anti-tubulin (catalog T9026, MilliporeSigma, 1:2000). After washing in TBS-Tween, blots were incubated with anti-rabbit, anti-mouse, or anti-goat horseradish peroxidase-conjugated secondary antibodies (catalog A6154, catalog A9044, catalog AP180P, MilliporeSigma, 1:5000 to 1:20000) and developed with Super Signal West Pico chemiluminescent substrate (cata$\log 34580$, Life Technologies) or incubated with anti-rabbit or anti-mouse infrared dye-conjugated secondary antibody (FE3680210, FE30926210, LiCor, 1:5000 to 1:20000) for 1 hour at room temperature. Images were acquired on Odyssey FC Imaging System (LiCor), and bands were quantified by densitometric analysis using the Image Studio Lite 5.0 software (LiCor). Tubulin or total protein load (Ponceau red staining) was used as sample loading control. In in vitro experiments results are expressed in arbitrary units.

Immunofluorescence analyses in vitro. Podocytes were washed in PBS, fixed with $4 \%$ paraformaldehyde (catalog 157-8, Electron Microscopy Sciences) for 10 minutes, and permeabilized by $0.5 \%$ Triton X-100 (catalog 93418, MilliporeSigma) for 30 or 10 minutes at room temperature for TR $\alpha 1$ or DIO3 staining, respectively. After washing with PBS and blocking of nonspecific binding sites with 3\% BSA (catalog A7906, MilliporeSigma) for 45 minutes at room temperature, cells were incubated with primary rabbit antiTR $\alpha 1$ antibody (catalog PA1-211A, Invitrogen, 1:80) or rabbit anti-DIO3 (catalog NBP1-05767, Novus Biologicals, 1:80) diluted in 1\% BSA, overnight at $4^{\circ} \mathrm{C}$. After washing in PBS, cells were incubated with donkey anti-rabbit Cy3-conjugated secondary antibody (catalog 115-096-072, Jackson ImmunoResearch Laboratories, 1:50) for 1 hour at room temperature. For F-actin staining, podocytes were incubated with FITC-conjugated phalloidin (catalog A12389, Life Technologies, 1:150) for 45 minutes at room temperature. Nuclei were stained with DAPI (catalog D9542, MilliporeSigma, $1 \mu \mathrm{g} / \mathrm{ml}$ ). Slides were mounted with Dako fluorescence mounting medium (catalog S3023, MilliporeSigma). Negative controls were obtained by omitting primary antibodies. Digital images were acquired using an inverted confocal laser microscope (Leica TCS SP8, Leica Biosystems) or Apotome Axio Imager Z2 (Zeiss). Images were analyzed with LAS $\mathrm{X}$ Industry Software and ImageJ. To quantify the number of cells with peripheral F-actin distribution, 15 random images for each sample were acquired and the percentage of cells with F-actin rearrangement with respect to total cells for each field was then evaluated in blind fashion.

Cell hypertrophy. Podocyte hypertrophy was measured as a ratio between total protein content and cell number, as described previously (73). Data are expressed in arbitrary units. 
Cell cycle analysis. Cell cycle perturbations were analyzed at the end of treatments using the Allophycocyanin Bromodeoxyuridine (BrdU) Flow Kit (catalog 552598; BD Biosciences) following the manufacturer's instructions. Briefly, cells were incubated with BrdU for 30 minutes in a humidified incubator and then harvested. After fixing and permeabilizing, cells were then treated with $300 \mu \mathrm{g} / \mathrm{ml} \mathrm{DNase}$ for 1 hour at $37^{\circ} \mathrm{C}$, and immunofluorescent staining with anti-BrdU antibody was performed. DNA was stained with 7-amino-actinomycin D. Finally, samples were analyzed by BD LSRFortessa X-20 (BD Biosciences) and acquired using the FACSDiVa software (BD Biosciences). At least 10,000 events per samples were analyzed using the FlowJo software.

$S A-\beta$-Gal activity assay. SA- $\beta$-Gal activity was measured with a Senescence Detection Kit (catalog ab65351, Abcam), according to the manufacturer's instructions. Briefly, cells were fixed for 15 minutes at room temperature and then stained with a solution containing $\mathrm{X}-\mathrm{Gal}$ at $37^{\circ} \mathrm{C}$ overnight. During incubation, plates were put inside resealable bags. The next day, staining solution was removed and cells were overlaid with 70\% glycerol. Images were captured using an inverted microscope (Primo Vert, Carl Zeiss) equipped with the AxioCam ERc 5s Rev. 2.0.

Statistics. Statistical analysis was performed using Graph Pad Prism software. All data are expressed as mean \pm SEM and in scatter dot plot diagrams. Comparisons were made using 2-tailed unpaired Student's $t$ test or 1-way ANOVA with Tukey's multiple-comparisons post hoc test as appropriate. Correlations between $\mathrm{T}_{3}$ levels and hallmark of metabolic/renal disease were determined by linear regression. Statistical significance was set at $P<0.05$. See figure legends for more details on statistical tests and $n$ values used.

Study approval. All procedures involving animals were performed with the approval of and in accordance with internal institutional guidelines of the Istituto di Ricerche Farmacologiche Mario Negri IRCCS, which are in compliance with national (D.L.n.26, March 4, 2014) and international laws and policies (directive 2010/63/EU on the protection of animals used for scientific purposes).

Renal tissues of patients $(n=3)$ with $\mathrm{DN}$ from the archives of the Unit of Nephrology, Azienda Azienda Socio Sanitaria Territoriale (ASST) Papa Giovanni XXIII (Bergamo, Italy) were studied. Specimens of uninvolved portions of kidney collected from tumor nephrectomy were used as controls. All experimental protocols involving human subjects and requiring informed consent were carried out in accordance with the Declaration of Helsinki and good clinical practise guidelines and were approved by the ethical committee of the ASST Papa Giovanni XXIII.

\section{Author contributions}

V. Benedetti carried out in vitro experiments; collected, analyzed, and interpreted the data; prepared the figures; and prepared the manuscript. AML carried out in vitro experiments; performed Western blot and immunohistochemistry experiments on animals; collected, analyzed, and interpreted the data; and prepared the figures. ML performed immunohistochemistry experiments on animals and patients. V. Brizi carried out in vitro experiments, performed immunohistochemistry experiments in animals and cultured cells, and was responsible for the SA- $\beta$-Gal activity assay. DC performed animal studies. MT performed FACS analysis. RN performed immunohistochemistry experiments in patients and wrote the manuscript. $A B$ edited and approved the final version of the manuscript. CZ supervised the in vivo studies and approved the final version of the manuscript. GR critically revised the manuscript and approved the final version of the manuscript. CX conceived and designed the experiments, analyzed and interpreted data, wrote the manuscript, and supervised the study. All authors reviewed the manuscript.

\section{Acknowledgments}

The authors are grateful to Kerstin Mierke for the excellent editing work on the manuscript and Sara Buttò for technical contributions to the in vitro studies. Valentina Benedetti, Angelo Michele Lavecchia, and Rubina Novelli are recipients of fellowships from Fondazione Aiuti per la Ricerca sulle Malattie Rare, Bergamo, Italy. This research was partially funded by the European Foundation for the Study of Diabetes/ Novo Nordisk and the Associazione per la Ricerca sul Diabete Italia.

Address correspondence to: Christodoulos Xinaris, Istituto di Ricerche Farmacologiche Mario Negri IRCCS, Centro Anna Maria Astori, Science and Technology Park Kilometro Rosso, Department of Molecular Medicine, Via Stezzano, 87, 24126 Bergamo, Italy. Phone: 39.035.42131; Email: christodoulos. xinaris@marionegri.it. 
1. Taylor E, Heyland A. Evolution of thyroid hormone signaling in animals: Non-genomic and genomic modes of action. Mol Cell Endocrinol. 2017;459:14-20.

2. Mourouzis I, Lavecchia AM, Xinaris C. Thyroid hormone signalling: from the dawn of life to the bedside [published online ahead of print August 27, 2019]. J Mol Evol. doi: 10.1007/s00239-019-09908-1.

3. Little AG, Seebacher F. The evolution of endothermy is explained by thyroid hormone-mediated responses to cold in early vertebrates. J Exp Biol. 2014;217(Pt 10):1642-1648.

4. Brent GA. Mechanisms of thyroid hormone action. J Clin Invest. 2012;122(9):3035-3043.

5. Heyland A, Reitzel AM, Hodin J. Thyroid hormones determine developmental mode in sand dollars (Echinodermata: Echinoidea). Evol Dev. 2004;6(6):382-392.

6. Laudet V. The origins and evolution of vertebrate metamorphosis. Curr Biol. 2011;21(18):R726-R737.

7. Forhead AJ, Fowden AL. Thyroid hormones in fetal growth and prepartum maturation. J Endocrinol. 2014;221(3):R87-R103.

8. Mai W, et al. Thyroid hormone receptor alpha is a molecular switch of cardiac function between fetal and postnatal life. Proc Natl Acad Sci USA. 2004;101(28):10332-10337.

9. Horn S, Heuer H. Thyroid hormone action during brain development: more questions than answers. Mol Cell Endocrinol. 2010;315(1-2):19-26.

10. Santini F, et al. Serum iodothyronines in the human fetus and the newborn: evidence for an important role of placenta in fetal thyroid hormone homeostasis. J Clin Endocrinol Metab. 1999;84(2):493-498.

11. Wu J, Li X, Tao Y, Wang Y, Peng Y. Free Triiodothyronine Levels Are Associated with Diabetic Nephropathy in Euthyroid Patients with Type 2 Diabetes. Int J Endocrinol. 2015;2015:204893.

12. Zou J, et al. Association between Thyroid Hormone Levels and Diabetic Kidney Disease in Euthyroid Patients with Type 2 Diabetes. Sci Rep. 2018;8(1):4728.

13. Wu P. Thyroid disorders and diabetes. It is common for a person to be affected by both thyroid disease and diabetes. Diabetes Self Manag. 2007;24(5):80-82.

14. Zoccali C, Mallamaci F, Tripepi G, Cutrupi S, Pizzini P. Low triiodothyronine and survival in end-stage renal disease. Kidney Int. 2006;70(3):523-528.

15. Rhee CM. The interaction between thyroid and kidney disease: an overview of the evidence. Curr Opin Endocrinol Diabetes Obes. 2016;23(5):407-415.

16. Lazzeri C, Sori A, Picariello C, Chiostri M, Gensini GF, Valente S. Nonthyroidal illness syndrome in ST-elevation myocardial infarction treated with mechanical revascularization. Int J Cardiol. 2012;158(1):103-104.

17. Najafian B, Alpers CE, Fogo AB. Pathology of human diabetic nephropathy. Contrib Nephrol. 2011;170:36-47.

18. Mukhi D, Nishad R, Menon RK, Pasupulati AK. Novel Actions of Growth Hormone in Podocytes: Implications for Diabetic Nephropathy. Front Med (Lausanne). 2017;4:102.

19. Lin JS, Susztak K. Podocytes: the Weakest Link in Diabetic Kidney Disease? Curr Diab Rep. 2016;16(5):45.

20. Herman-Edelstein M, Thomas MC, Thallas-Bonke V, Saleem M, Cooper ME, Kantharidis P. Dedifferentiation of immortalized human podocytes in response to transforming growth factor- $\beta$ : a model for diabetic podocytopathy. Diabetes. 2011;60(6):1779-1788.

21. Kriz W, Hähnel B, Rösener S, Elger M. Long-term treatment of rats with FGF-2 results in focal segmental glomerulosclerosis. Kidney Int. 1995;48(5):1435-1450.

22. Lasagni L, Lazzeri E, Shankland SJ, Anders HJ, Romagnani P. Podocyte mitosis - a catastrophe. Curr Mol Med. 2013;13(1):13-23.

23. Kato H, et al. Wnt/ $\beta$-catenin pathway in podocytes integrates cell adhesion, differentiation, and survival. $J$ Biol Chem. 2011;286(29):26003-26015.

24. Sweetwyne MT, Gruenwald A, Niranjan T, Nishinakamura R, Strob1 LJ, Susztak K. Notch1 and Notch2 in Podocytes Play Differential Roles During Diabetic Nephropathy Development. Diabetes. 2015;64(12):4099-4111.

25. Niranjan T, et al. The Notch pathway in podocytes plays a role in the development of glomerular disease. Nat Med. 2008;14(3):290-298.

26. Li Y, Kang YS, Dai C, Kiss LP, Wen X, Liu Y. Epithelial-to-mesenchymal transition is a potential pathway leading to podocyte dysfunction and proteinuria. Am J Pathol. 2008;172(2):299-308.

27. Herman-Edelstein M, Thomas MC, Thallas-Bonke V, Saleem M, Cooper ME, Kantharidis P. Dedifferentiation of immortalized human podocytes in response to transforming growth factor- $\beta$ : a model for diabetic podocytopathy. Diabetes. 2011;60(6):1779-1788.

28. Harder JL, et al. Organoid single cell profiling identifies a transcriptional signature of glomerular disease. JCI Insight. 2019;4(1):122697.

29. Dressler GR, Deutsch U, Chowdhury K, Nornes HO, Gruss P. Pax2, a new murine paired-box-containing gene and its expression in the developing excretory system. Development. 1990;109(4):787-795.

30. Kobayashi A, et al. Six2 defines and regulates a multipotent self-renewing nephron progenitor population throughout mammalian kidney development. Cell Stem Cell. 2008;3(2):169-181.

31. Qiao J, Sakurai H, Nigam SK. Branching morphogenesis independent of mesenchymal-epithelial contact in the developing kidney. Proc Natl Acad Sci USA. 1999;96(13):7330-7335.

32. Loeffler I, Wolf G. Epithelial-to-Mesenchymal Transition in Diabetic Nephropathy: Fact or Fiction? Cells. 2015;4(4):631-652.

33. Tsui CC, Shankland SJ, Pierchala BA. Glial cell line-derived neurotrophic factor and its receptor ret is a novel ligand-receptor complex critical for survival response during podocyte injury. $J$ Am Soc Nephrol. 2006;17(6):1543-1552.

34. Miyauchi M, et al. Hypertrophy and loss of podocytes in diabetic nephropathy. Intern Med. 2009;48(18):1615-1620.

35. Dai H, Liu Q, Liu B. Research Progress on Mechanism of Podocyte Depletion in Diabetic Nephropathy. J Diabetes Res. 2017;2017:2615286

36. Bernal J, Morte B. Thyroid hormone receptor activity in the absence of ligand: physiological and developmental implications. Biochim Biophys Acta. 2013;1830(7):3893-3899.

37. Burton DGA, Faragher RGA. Obesity and type-2 diabetes as inducers of premature cellular senescence and ageing. Biogerontology. 2018;19(6):447-459.

38. Verzola D, et al. Accelerated senescence in the kidneys of patients with type 2 diabetic nephropathy. Am J Physiol Renal Physiol. 2008;295(5):F1563-F1573. 
39. Dimri GP, et al. A biomarker that identifies senescent human cells in culture and in aging skin in vivo. Proc Natl Acad Sci USA. 1995;92(20):9363-9367.

40. Pantos C, Xinaris C, Mourouzis I, Malliopoulou V, Kardami E, Cokkinos DV. Thyroid hormone changes cardiomyocyte shape and geometry via ERK signaling pathway: potential therapeutic implications in reversing cardiac remodeling? Mol Cell Biochem. 2007;297(1-2):65-72.

41. Mourouzis I, Kostakou E, Galanopoulos G, Mantzouratou P, Pantos C. Inhibition of thyroid hormone receptor $\alpha 1$ impairs post-ischemic cardiac performance after myocardial infarction in mice. Mol Cell Biochem. 2013;379(1-2):97-105.

42. Ganesan K, Wadud K. Euthyroid Sick Syndrome. In: StatPearls. Treasure Island, Florida, USA: StatPearls Publishing; 2019.

43. Pantos C, Mourouzis I, Xinaris C, Papadopoulou-Daifoti Z, Cokkinos D. Thyroid hormone and "cardiac metamorphosis": potential therapeutic implications. Pharmacol Ther. 2008;118(2):277-294.

44. Blanco-Molina A, González-Reyes JA, Torre-Cisneros J, López-Miranda J, Nicolás M, Pérez-Jiménez F. Effects of hypothyroidism on the ultrastructure of rat pancreatic acinar cells: a stereological analysis. Histol Histopathol. 1991;6(1):37-42.

45. Nam SM, et al. Hypothyroidism affects astrocyte and microglial morphology in type 2 diabetes. Neural Regen Res. 2013;8(26):2458-2467.

46. Vadászová A, Zacharová G, Machácová K, Jirmanová I, Soukup T. Influence of thyroid status on the differentiation of slow and fast muscle phenotypes. Physiol Res. 2004;53 Suppl 1:S57-S61.

47. Charalambous M, Hernandez A. Genomic imprinting of the type 3 thyroid hormone deiodinase gene: regulation and developmental implications. Biochim Biophys Acta. 2013;1830(7):3946-3955.

48. Bouzaffour M, Rampon C, Ramaugé M, Courtin F, Vriz S. Implication of type 3 deiodinase induction in zebrafish fin regeneration. Gen Comp Endocrinol. 2010;168(1):88-94.

49. Boelen A, et al. Induction of type 3 deiodinase activity in inflammatory cells of mice with chronic local inflammation. Endocrinology. 2005;146(12):5128-5134.

50. Boelen A, et al. Type 3 deiodinase is highly expressed in infiltrating neutrophilic granulocytes in response to acute bacterial infection. Thyroid. 2008;18(10):1095-1103.

51. Dentice M, Antonini D, Salvatore D. Type 3 deiodinase and solid tumors: an intriguing pair. Expert Opin Ther Targets. 2013;17(11):1369-1379.

52. Kester MH, et al. Large induction of type III deiodinase expression after partial hepatectomy in the regenerating mouse and rat liver. Endocrinology. 2009;150(1):540-545.

53. Janssen R, Zuidwijk M, Muller A, Mulders J, Oudejans CB, Simonides WS. Cardiac expression of deiodinase type 3 (Dio3) following myocardial infarction is associated with the induction of a pluripotency microRNA signature from the Dlk1-Dio3 genomic region. Endocrinology. 2013;154(6):1973-1978.

54. Pantos C, et al. Thyroid hormone receptor alpha 1: a switch to cardiac cell "metamorphosis"? J Physiol Pharmacol. 2008;59(2):253-269

55. Pantos C, Xinaris C, Mourouzis I, Malliopoulou V, Kardami E, Cokkinos DV. Thyroid hormone changes cardiomyocyte shape and geometry via ERK signaling pathway: potential therapeutic implications in reversing cardiac remodeling? Mol Cell Biochem. 2007;297(1-2):65-72.

56. Pantos C, et al. Thyroid hormone attenuates cardiac remodeling and improves hemodynamics early after acute myocardial infarction in rats. Eur J Cardiothorac Surg. 2007;32(2):333-339.

57. Mourouzis I, et al. Thyroid hormone improves the mechanical performance of the post-infarcted diabetic myocardium: a response associated with up-regulation of Akt/mTOR and AMPK activation. Metab Clin Exp. 2013;62(10):1387-1393

58. Cokkinos DV, Pantos C. [Type 1 diabetes impairs compensatory response after myocardial infarction; role of tissue hypothyroidism and effects of thyroid hormone administration]. Bull Acad Natl Med. 2011;195(1):151-164.

59. Kalofoutis C, et al. Thyroid hormone can favorably remodel the diabetic myocardium after acute myocardial infarction. Mol Cell Biochem. 2010;345(1-2):161-169.

60. Lourbopoulos A, et al. Changes in thyroid hormone receptors after permanent cerebral ischemia in male rats. JMol Neurosci. 2014;54(1):78-91.

61. Milanesi A, et al. Thyroid Hormone Receptor Alpha is Essential to Maintain the Satellite Cell Niche During Skeletal Muscle Injury and Sarcopenia of Aging. Thyroid. 2017;27(10):1316-1322.

62. Rodríguez-Gómez I, et al. Influence of thyroid state on cardiac and renal capillary density and glomerular morphology in rats J Endocrinol. 2013;216(1):43-51.

63. Pantos C, et al. Propylthiouracil-induced hypothyroidism is associated with increased tolerance of the isolated rat heart to ischaemia-reperfusion. J Endocrinol. 2003;178(3):427-435.

64. Sekimizu K, Tagawa M, Takeda H. Defective fin regeneration in medaka fish (Oryzias latipes) with hypothyroidism. Zool Sci. 2007;24(7):693-699.

65. Collet TH, et al. Subclinical hyperthyroidism and the risk of coronary heart disease and mortality. Arch Intern Med. 2012;172(10):799-809.

66. Dousdampanis P, Trigka K, Vagenakis GA, Fourtounas C. The thyroid and the kidney: a complex interplay in health and disease. Int J Artif Organs. 2014;37(1):1-12.

67. Ali Rajab NM, Ukropina M, Cakic-Milosevic M. Histological and ultrastructural alterations of rat thyroid gland after shortterm treatment with high doses of thyroid hormones. Saudi J Biol Sci. 2017;24(6):1117-1125.

68. Pantos C, Mourouzis I. Thyroid hormone receptor $\alpha 1$ as a novel therapeutic target for tissue repair. Ann Transl Med. 2018;6(12):254

69. Cassis P, et al. ADAMTS13 Deficiency Shortens the Life Span of Mice With Experimental Diabetes. Diabetes. 2018;67(10):2069-2083.

70. Macconi D, et al. Pathophysiologic implications of reduced podocyte number in a rat model of progressive glomerular injury. Am J Pathol. 2006;168(1):42-54.

71. Fukuda A, et al. Growth-dependent podocyte failure causes glomerulosclerosis. J Am Soc Nephrol. 2012;23(8):1351-1363.

72. Saleem MA, et al. A conditionally immortalized human podocyte cell line demonstrating nephrin and podocin expression. J Am Soc Nephrol. 2002;13(3):630-638.

73. Pantos C, et al. Time-dependent changes in the expression of thyroid hormone receptor alpha 1 in the myocardium after acute myocardial infarction: possible implications in cardiac remodelling. Eur J Endocrinol. 2007;156(4):415-424. 